\title{
Pre-electoral Coalitions and Post-election Bargaining ${ }^{1}$
}

\author{
Siddhartha Bandyopadhyay Kalyan Chatterjee Tomas Sjöström
}

September 15, 2009

${ }^{1}$ We thank Facundo Albornoz, Ralph Bailey, Jayasri Dutta, John Fender, Indridi Indridason, Saptarshi Ghosh and Sona Golder for valuable comments. 


\begin{abstract}
Pre-electoral coalitions occur frequently in parliamentary democracies. They influence post election coalition formation and surplus division. We study a game theoretic model where political parties can form coalitions both before (ex ante) and after (ex post) the elections. Ex ante coalitions can commit to a seat-sharing arrangement, but neither to a policy nor to a division of rents from office; coalition members are even free to break up and join other coalitions after the election. Equilibrium ex ante coalitions are not necessarily made up of the most ideologically similar parties, and they form under (national list) proportional representation as well as plurality rule. They do not form just to avoid "splitting the vote", but also because seat-sharing arrangements will influence the ex post bargaining and coalition formation. The ex post bargaining protocol matters greatly: there is more scope for coalition formation, both ex ante and ex post, under an Austen-Smith and Banks protocol than under "random recognition".
\end{abstract}




\section{Introduction}

In parliamentary democracies, coalition governments are common, and single party majority governments are relatively rare. A study of 313 elections in 11 European democracies between 1945 and 1997 found that only 20 elections returned a single party with more than half of all seats in parliament (Gallagher, Laver and Mair, 1995, Diermeier and Merlo, 2004). But coalitions can form both ex ante (before elections) and ex post (after elections). In a study of 364 elections in 23 advanced parliamentary democracies between 1946 and 2002, Golder (2006 a and b) found 240 instances of pre-electoral agreements. Such agreements are common in diverse countries such as France, South Korea and India. Debus (2009) offers empirical evidence that pre-electoral alliances have an impact on government formation. Yet the theoretical literature on the subject is not large. In this paper, we investigate how different electoral systems and post-election bargaining protocols influence equilibrium coalition formation.

It is well known that under plurality voting (PV), like-minded parties may end up "splitting the vote" by competing against each other in the same electoral districts. In 1903, the U.K. Labour party and Liberal Democrats formed the first "Lib-Lab" pact, wherein they agreed not to compete against each other for 50 seats in parliament (Pugh, 2002, p. 117). Various forms of Lib-Lab arrangements persist to this day, mainly in local elections in Scotland and Wales, though attempts at national seat-sharing agreements have also been made. In India, which also has a PV electoral system, pre-election coalitions became widespread following the 1977 election, when the Indian National Congress lost its hold on power. ${ }^{1}$ These pre-electoral agreements often do not involve a commitment to a set of policies, or to forming a coalition government. The main issue over which the various pre-electoral alliances in India bargain is which party will contest which seat.

Under a system of proportional representation (PR) with national lists, as in Israel, each list gets a number of seats in parliament proportional to its vote share. If two parties stand on a joint list, and if each voter who supports either party votes for the joint list, then the joint list will get the same number of seats in parliament as the two parties would get by standing on separate lists. Thus, in this system, the problem of splitting the vote is moot, seemingly eliminating the rationale for ex ante agreements. But in reality, ex ante coalitions occur even with proportional representation. For example, 87\% of the elections in Israel (which comes closest to a "pure" form of PR) analyzed in Golder's data set had at least one pre-electoral alliance. Similarly, joint lists have been seen in Greece, Portugal and (to

\footnotetext{
${ }^{1}$ See http://www.electionresults.in/history-political-parties.html for a brief history of how the Indian national Congress lost its hold on power.
} 
a lesser extent) Netherlands (see again Golder 2006 a, 2006 b for details). Our theoretical model investigates the possible motives for such pre-electoral agreements.

In our model there are three parties, L,M and R, with M ideologically closer to L than to R. The parties care about ideology, "rents from office" and seats in parliament. If ideologically distant parties form a coalition government, they may experience costs of ideological compromises. Therefore, an MR coalition government (made up of the M and R parties) generates a smaller surplus than an LM government. (For simplicity, we assume the L and R parties are so far apart ideologically that a coalition between them cannot generate any surplus). In addition, if a party is outside the government, it may suffer a negative externality from a government to which it is ideologically opposed.

Most real-world PR systems are characterized by a combination of national list choice and district level elections. However, in order to isolate the "splitting-the-vote" motive for ex ante coalitions, we will study a "pure" system of strictly proportional representation with national lists where this motive is absent. Under this voting system, an ex ante coalition is simply an agreement to contest the election as a single national list. The ordering of candidates on the list will determine the parties' vote shares and hence seats in parliament. We also study a second voting system, plurality voting (PV), where the electorate is divided into districts and each district elects a member of parliament. Under this voting system, an ex ante coalition is an agreement not to compete against each other in certain districts. This may not be a complete seat-sharing arrangement; there may be some seats in which both parties run for office. ${ }^{2}$

In our model, ex ante coalitions determine the seat shares of the coalition partners, but they are free to split up after the election. If no party obtains a majority of the seats in parliament, then post-election bargaining determines which government forms, and how the rents from office are allocated. This stark model is meant to explore the "pure" incentives for coalition formation. It abstracts from issues such as increasing returns to scale in campaign effort, which would make an ex ante coalition more profitable, and instead focuses on the role of ex post bargaining and coalition formation.

The incentives to form ex ante coalitions are influenced by the ex post bargaining protocol. To investigate this point, we consider two "canonical" ex post bargaining protocols. The "random recognition protocol" specifies that, in each "round" of bargaining, each party is recognized to propose a coalition with probability proportional to its number of seats in parliament. Similar protocols have been analyzed by Baron and Ferejohn, 1989, and

\footnotetext{
${ }^{2}$ For example, in 2001 in Assam (one of the states in India), the BJP and AGP parties agreed that the BJP would put up candidates for 44 seats, but 10 of these would be contested by both parties in "friendly contests" (see http://news.indiamart.com/news-analysis/assembly-polls-congr-6008.html).
} 
others. The ASB protocol (named for Austen-Smith and Banks, 1988) instead specifies that the largest party is always recognized first, followed by the second largest and so on. We characterize the stationary subgame perfect equilibria of the infinite-horizon ex post bargaining games corresponding to the two protocols. With random recognition, $\mathrm{M}$ and $\mathrm{R}$ never form a coalition government. Equilibrium surplus shares within the governing coalition are proportional to seat shares, though M's payoff is bounded below. With the ASB protocol, $\mathrm{M}$ and $\mathrm{R}$ can form a coalition government ex post, as long as they are not too ideologically distant, and as long as $\mathrm{R}$ is small enough (making it an attractive coalition partner for $\mathrm{M}$ ).

There are three motives for ex ante coalitions in our model: (a) to influence which government will form ex post; (b) to manipulate the bargaining power within the government; and (c) with plurality voting only, similar parties avoid splitting the vote. We emphasize (a) and (b), as (c) is well known (e.g., Golder, 2006 a, 2006 b and Blais and Indridason, 2007 who examine this motive in the context of runoffs). One way for motive (a) to come about is via an ex ante agreement which produces such a large vote share for $M$ that it becomes a majority party. The "junior" ex ante coalition partner, say R, benefits from this seemingly one-sided agreement because it blocks its ideological opponent $\mathrm{L}$ from joining a coalition government. If the Austen-Smith and Banks protocol operates ex post, then there is another way for (a) to happen: the junior ex ante coalition partner, say R, transfers enough seats to $\mathrm{M}$ so that, even if $\mathrm{M}$ does not get its own majority, $\mathrm{R}$ becomes so small that $\mathrm{M}$ finds $\mathrm{R}$ an attractive coalition partner ex post. Motive (b) can come about via an ex ante agreement that transfers enough vote shares to change the ex post distribution of surplus, via the ex post bargaining protocol, without actually changing the governing coalition. Because of (a) and (b), ex ante agreements may be viable under PR. Also, because of these motives, under PV ideologically different parties (M and R) unconcerned about "splitting the vote" may still find a viable ex ante agreement. Thus, one of our main conclusions is that, in theory at least, ex ante coalitions are by no means motivated solely by the problem of "splitting the vote". Finally, we show that strategic voting cannot replicate the outcomes that are induced by pre-electoral seat sharing arrangements.

There is a large game theoretic literature on bargaining and coalition formation. A sequential, proposal-making model of coalition formation with transferable utility is analyzed by Chatterjee, Dutta, Ray and Sengupta (1993). Okada (1996, 2007) considers a similar model for superadditive games, where the proposers are randomly selected among the remaining set of players after any rejection. Eraslan and Merlo (2002) analyze a random proposer model in which only one coalition forms. In their model there is only one "pie", whose size could vary randomly over time but which can only be consumed if a majority of players (or other quota) decides to do so. Ray (2008) surveys these and other models, 
including ones with externalities. Our random recognition protocol does not require the game to be superadditive, there are externalities, and different coalitions generate different surpluses. Our sequential proposal-making (ASB) protocol was inspired by Austen Smith and Banks (1988).

Starting with Riker (1962), a large literature in political science discusses coalition formation in legislatures. ${ }^{3}$ Riker's concern was with sharing a fixed pie (the rents from government). Axelrod (1970) added ideological motives. Austen Smith and Banks (1988) provide a formal game-theoretic model of how the nature of coalitions (ex post) influence voting. Diermeier and Merlo (2000) and Baron and Diermeier (2001) study post-election coalitional diversity. Indridason $(2003,2005)$ empirically studies what factors affect the size and connectedness of coalitions and Bandyopadhyay and Oak (2004, 2008) develop a theoretical model. All these models ignored pre-electoral coalitions. This gap was remedied by Golder (2006 a, 2006 b), who offered both a theoretical model and an empirical analysis. Our theoretical model differs from Golder's in several ways. First, we model political competition explicitly: parties have a choice of coalition partners. In Golder's model, the identity of the coalition partner is not a choice variable (the choice is only whether to accept this partner or not). Second, we explicitly model the voting process. Third, Golder assumes pre-electoral coalitions make binding commitments on policy and rents from office. In our model, pre-electoral coalitions agree on seat-sharing arrangements, but make no other commitments (on future policies, surplus-sharing or government formation).

We study how a particular kind of partial commitment, namely seat-sharing arrangements, can be used to influence ex post coalition formation and surplus division. While there is no agreement in the literature about what parties can commit to, the perfect commitment assumption of the Downsian model (Downs, 1957) is often viewed as unrealistic. Models such as the citizen candidate models (Osborne and Slivinski 1996, Besley and Coate, 1997) assume no commitment. In our context, if commitment were perfect there would be no need to use seat-sharing arrangements to indirectly influence ex post outcomes, because these could be contracted on directly. The only motive for seat-sharing arrangements would then be to avoid splitting the vote under PV. However, as pointed out by Debus (2009) and Golder (2006 a and b), pre-electoral coalitions also influence the nature of coalitions that form post election. Moreover, pre-electoral alliances often do break up, with former coalition partners not cooperating in forming a government, suggesting less than perfect commitment. For example, the Janata Party, a merger of various groups opposed to the Congress, won the national election in India in 1977. After a few years, the Janata Party split into its com-

\footnotetext{
${ }^{3}$ See Laver and Schofield (1990) and Roemer (2001). For a survey, see Bandyopadhyay and Chatterjee (2006).
} 
ponents, and these have since formed a number of pre-electoral coalitions. These coalitions are clearly not mergers; the parties consider themselves free to join other coalitions ex post. For example, The Hindu newspaper of May 15, 2009 reported that Nitish Kumar of the Janata Dal (United) party, a member of the pre-electoral coalition "National Democratic Alliance", stated his conditions for supporting any coalition government, possibly one not formed by the National Democratic Alliance. Several members of the pre-electoral alliance "Third Front" also declared themselves ready to switch to other groupings after the election.

With perfect commitment, ex ante agreements would be akin to a forming a new party. Dhillon (2005) surveys the party formation literature. In a seminal contribution, Morelli (2004) assumed new parties form by mergers of old parties. These mergers involve binding commitments on policy and ex post cooperation. ${ }^{4}$ In our model, a pre-electoral coalition does not signify a "merger" where the parties give up their separate identities. Instead, the parties remain independent and (as long as no party has its own majority) must bargain ex post to form a coalition government. In addition, unlike in Morelli (2004), our parties get utility not only from seats in parliament, but also from joining the government, and even from blocking ideologically distant parties from joining. The issue of maintaining separate identities versus mergers is also analyzed by Persson, Roland and Tabellini (2007). Their parties (unlike ours) are opportunistic and represent specific constituencies and not ideological positions, and their focus is on comparing government spending under single party versus coalition governments.

The rest of the paper proceeds as follows. In section 2 we present the model. Section 3 considers post-election bargaining under the two protocols. Sections 4 and 5 analyze ex ante coalitions under PR and PV, respectively. Section 6 briefly discusses strategic voting. Section 7 concludes.

\section{The Model}

\subsection{Parties, Voters and Preferences}

There are three parties arranged from left to right, $L, M$ and $R$. There are three kinds of voters: $L$-supporters, $M$-supporters, and $R$-supporters. Voter preferences are single-peaked in the sense that $L$-supporters rank party $L$ first, party $M$ second and party $R$ last, and $R$-supporters rank $R$ first, $M$ second and $L$ last. Without loss of generality, we assume the $M$ party is ideologically closer to the $L$ party, so the $M$-supporters rank $M$ first, $L$ second

\footnotetext{
${ }^{4}$ Other important work on party formation includes Roemer (2001), Jackson and Moselle (2002), Snyder and Ting (2002), Levy (2004) and Osborne and Tourky (2002).
} 
and $R$ last. Let $v(P)$ denote the fraction of all voters who support party $P \in\{L, M, R\}$. To avoid trivialities we assume $0<v(P)<1 / 2$ for each $P \in\{L, M, R\}$. Party $P$ 's share of the seats in parliament is denoted $n(P)$, where $n(L)+n(M)+n(R)=1$. For convenience, we normalize the total number of seats in parliament to equal 1, so that a party's number of seats equals its share of the seats. We assume voters vote sincerely (but we briefly discuss strategic voting in section 6 ). This means, $P$-supporters vote for party $P$ whenever possible. Their behavior when this is not possible (because of an ex ante coalition) is discussed below.

Each party is considered an individual player who derives utility from seats in parliament. Let $\alpha$ denote the value of a seat, which is the same for all parties. In addition, if a party is a member of government, it enjoys a share of the surplus generated by the government, the "rents from office". Parties also care about policy, for two reasons: (i) they face a compromise cost if they form a coalition, the compromise cost being lower if the partners are ideologically closer; and (ii) if they are not in government then they suffer a cost from the policy implemented by the party (or parties) in government, the cost being lower if the government is ideologically closer to them. A one-party government generates a net surplus $S$. A two party coalition government consisting of parties $P$ and $P^{\prime}$ generates a smaller surplus $S\left(P, P^{\prime}\right)<S$ due to costly compromises. The compromise cost is greater (hence the surplus is smaller), the more ideologically distant are the two parties, so $S(M, R)<S(L, M)$. To simplify, we assume $S(L, R)=0$ so parties $L$ and $R$ will never form a coalition government (and we avoid having too many special cases). Thus, we assume

$$
0=S(L, R)<S(M, R)<S(L, M)<S
$$

If party $P$ is part of the government then $s(P)$ denotes its (endogenously determined) share of the surplus. In the case of a one-party government, $s(P)=S$. For a two-party government, $s(P)+s\left(P^{\prime}\right)=S\left(P, P^{\prime}\right)$.

A government may impose negative externalities on outsiders (say, by implementing policies they don't like). Formally, if party $P$ is not a member of government, it suffers a $\operatorname{cost} x_{P}\left(P^{\prime}, P^{\prime \prime}\right)$ if the other two parties $P^{\prime}$ and $P^{\prime \prime}$ form a coalition government, and $x_{P}\left(P^{\prime}\right)$ if party $P^{\prime}$ forms a one party government. We assume

$$
0 \leq x_{P}(M)<x_{P}\left(M, P^{\prime}\right)
$$

for $P, P^{\prime} \neq M$. That is, each party $P \in\{L, R\}$ prefers a one-party $\mathrm{M}$ government to a coalition government where $\mathrm{M}$ governs with the other party $P^{\prime} \neq P$.

To summarize, if party $P$ is part of the government, then its payoff is $s(P)+\alpha n(P)$. If party $P$ is not part of the government, then its payoff is $\alpha n(P)-x_{P}$, where $x_{P}=x_{P}\left(P^{\prime}\right)$ if 
party $P^{\prime}$ forms a one-party government, and $x_{P}=x_{P}\left(P^{\prime}, P^{\prime \prime}\right)$ if $P^{\prime}$ and $P^{\prime \prime}$ form a coalition government. For example, if $M$ and $R$ form a two-party coalition, then the final payoff for $L$ will be $\alpha n(L)-x_{L}(M, R) .^{5}$

\subsection{Elections}

We consider two kinds of voting systems: proportional representation (PR) and plurality voting (PV). Proportional representation is a national election in which lists compete against each other. If all parties run for election on separate lists, and voting is sincere, then proportional representation implies $n(P)=v(P)$ for each $P \in\{L, M, R\}$.

To describe the outcome of plurality voting, we assume the electorate is divided into a large number of ex ante identical districts. We assume that the overall results of the election can be predicted with certainty ex ante, and this can be justified because the number of districts is assumed very large and there are no aggregate shocks. However, since the districts are ex ante identical, but experience idiosyncratic shocks to the election results, it is not possible to predict which particular districts will be won by which party. If all parties run for election in every district, then with sincere voting, party $P \in\{L, M, R\}$ wins a plurality in a fraction $w(P)$ of all districts, and a majority in a fraction $z(P)$ of all districts, where $0<z(P)<w(P)<1 / 2$. Under $\mathrm{PV}$, if all parties run in each district and voting is sincere, then $n(P)=w(P)$ for each $P \in\{L, M, R\}$. Note that $v(P) \neq w(P)$ in general. ${ }^{6}$ Also, to simplify and eliminate some less interesting cases, we assume it is not too likely a party wins a majority in any district. Specifically, we assume

$$
z(P)<\min \{w(L), w(M), w(R)\}
$$

for each $P \in\{L, M, R\}$.

\footnotetext{
${ }^{5}$ The compromise cost and externalities can be derived from a standard spatial framework, where parties in a coalition face a loss because the actual policy (arising out of some bargaining outcome within the coalition) differs from their ideal policy, and parties outside the coalition face a loss because of the same reason. (Only parties within the ruling coalition get a share of perks.)

${ }^{6}$ For example, suppose the districts are ex ante symmetrical, but when elections occur there is a random variable $x_{i}$ for district $i$ that takes one of three values ( $\mathrm{L}, \mathrm{M}$ or $\mathrm{R}$ ), each value occurring in a third of the districts. If $x_{i}=R$, L has support of $30 \%$ of the voters in district $i, \mathrm{M}$ has $20 \%$, and $\mathrm{R}$ has $50 \%$. If $x_{i}=L$ (resp. $x_{i}=M$ ) the numbers are $60 \%$ for L, 30\% for M, $10 \%$ for R (resp. 30\%, 40\%, 30\%).
}

$\begin{array}{llll} & .33 & .33 & .33 \\ \mathrm{~L} & .3 & .6 & .3 \\ \mathrm{M} & .2 & .3 & .4 \\ \mathrm{R} & .5 & .1 & .3\end{array}$

Here $w(P)=\frac{1}{3}$ for each party. However, the nationwide vote share is $v(L)=0.4, v(M)=v(R)=0.3$. 
If one party gets more than half of all seats in parliament, i.e., if $n(P)>1 / 2$ for some $P$, then party $P$ forms a one-party government and the game ends. If no party gets more than $50 \%$ of the seats, i.e., if $n(P) \leq 1 / 2$ for all $P$, then there will be post-election bargaining.

\subsection{Post-election Bargaining}

If no party has a majority of the seats in parliament, then two parties $P$ and $P^{\prime}$ can form a coalition government. Within the governing coalition, utility can be transferred (only) by allocating the surplus $S\left(P, P^{\prime}\right)$ the government generates. A proposal to form a coalition specifies how the surplus is to be shared. The most a party can offer a coalition partner is $100 \%$ of the surplus. ${ }^{7}$ A recognition rule or protocol determines the order in which proposals are made. Typically, the order is influenced by the election results: a larger party is more likely to be recognized to make a proposal. In this way, the elections influence the parties' ex post bargaining strength.

The post-election bargaining game has (potentially) an infinite number of periods, with discounting of future payoffs using a common discount factor $\delta$. As is standard, we will consider the limit as $\delta \rightarrow 1$. In period $t=1,2,3 \ldots$, party $P$ is chosen to make a proposal with probability $\xi_{P}(t)$. The function $\xi_{P}$ is called the recognition rule or protocol. The proposal is made to another party $P^{\prime}$, who responds by accepting or rejecting. If $P^{\prime}$ accepts then the game ends and the proposal is implemented. If $P^{\prime}$ rejects then the bargaining game moves to the next period. The infinite horizon specification is natural, since there is no natural pre-set deadline on post-election bargaining. The party who is recognized to make the very first proposal, at $t=1$, is called the formateur.

Different bargaining protocols exist in the literature. We consider two alternatives. In the first protocol, the biggest party (i.e., $P$ such that $n(P)>n\left(P^{\prime}\right)$ for all $P^{\prime} \neq P$ ) makes the first proposal, followed by the second biggest, etc. Formally, $\xi_{P}(t)=1$, if either $t=1,4,7$.. and $P$ is the party with the largest seat share, or $t=2,5,8 \ldots$ and $P$ is the party with the second largest seat share, or $t=3,6,9, \ldots$ and $P$ is the smallest party in terms of seat share. We call this the Austen-Smith and Banks (ASB) protocol. In the second protocol, the probability of being recognized in each period is directly proportional to the seat shares in parliament. Formally, $\xi_{P}(t)=n(P)$ for all $t$. We call this the random recognition protocol (cf. Baron and Ferejohn, 1989, Diermeier and Merlo, 2004). ${ }^{8}$

\footnotetext{
${ }^{7}$ Because of the negative externality, a player who does not become part of the government may get a negative payoff. However as the only way utility can be transferred is via the ex post surplus generated, a party is not allowed to offer another party more than $100 \%$ of the rents from office.

${ }^{8}$ In a working paper we also studied a sequential offers protocol, in which the rejector in period $t$ makes a proposal in period $t+1$. It did not generate any new insights so we do not discuss it here.
} 


\section{Equilibrium Post-Election Coalition Formation}

In this section we characterize the stationary subgame perfect equilibrium (SSPE) outcomes for the ASB and the random recognition protocols.

\subsection{The ASB protocol}

In the ASB protocol, the outcome of the elections fully determines the order of proposers. If $n\left(P^{\prime}\right)>n\left(P^{\prime \prime}\right)>n\left(P^{\prime \prime \prime}\right)$, then party $P^{\prime}$ makes the first proposal. If the proposal is rejected, $P^{\prime \prime}$ makes a proposal. If this is rejected, $P^{\prime \prime \prime}$ makes a proposal. If this is rejected, we go to the next "round", where again $P^{\prime}$ starts by making a proposal. Play continues until a proposal is accepted. Each proposal takes one "period", and a discount factor $\delta$ applies to each period. Periods 1,2,3 make up "round 1" periods 4,5,6 make up "round 2", etc. Each round uses the same fixed order $P^{\prime}, P^{\prime \prime}, P^{\prime \prime \prime}$. With a slight abuse of terminology, we call this ordering the bargaining protocol. In SSPE, defined for this protocol, stationarity means behavior in each round is independent of what happened in previous rounds.

Since the LR coalition is ruled out, $\mathrm{M}$ will either form a coalition with $\mathrm{L}$ or with $\mathrm{R}$. According to the ASB protocol, the largest party is the formateur (i.e., makes the very first proposal). Since $S(L, M)>S(M, R)$, if $\mathrm{L}$ is the formateur then $\mathrm{L}$ will always make an offer to $\mathrm{M}$ which is sufficient to get acceptance. The more interesting situation occurs when $\mathrm{L}$ is not the formateur. In this case, $\mathrm{M}$ may form a coalition with $\mathrm{R}$, even though $S(M, R)$ is smaller than $S(L, M)$, as long as (due to differences in bargaining strength) $\mathrm{M}$ gets a larger share of the surplus in the MR coalition than in the LM coalition. Intuitively, the "weaker" party is a more attractive coalition partner for $\mathrm{M}$, and so $\mathrm{M}$ and $\mathrm{R}$ may conclude their negotiations before $\mathrm{L}$ has a chance to make a proposal. Of course, the bargaining strength is determined by the bargaining protocol (which in turn is determined by the election results).

With ASB bargaining, the bargaining strength of $L$ vis-a-vis $M$ is captured by the number of periods which $\mathrm{L}$ has to wait to make an offer after rejecting an offer from M. Let $\lambda \in\{1,2\}$ denote this number. Thus, if M's proposal is rejected, then the next proposal is made by $\mathrm{L}$ if $\lambda=1$, but by $\mathrm{R}$ if $\lambda=2$. Notice that $\lambda$ is determined by the election results, e.g., if $n(M)>n(L)>n(R)$ then the bargaining protocol is $M L R$ so $\lambda=1$.

Party $\mathrm{L}$ is strong vis-a-vis $\mathrm{M}$ if $\lambda=1$, and this is the only case in which $\mathrm{R}$ has any hope of joining a coalition government. Formally, we have the following result.

Proposition 1 For $\delta$ close to 1, the ASB bargaining game has a unique SSPE outcome. The $M R$ coalition forms if $S(M, R)>\frac{1}{3} S(L, M)$ and the bargaining protocol is either $M L R$ or RML. Otherwise, the LM coalition forms. Whichever coalition forms, as $\delta \rightarrow 1$, M's 
share of the surplus converges to

$$
s^{\lambda}(M) \equiv \max \left\{\frac{\lambda}{3} S(L, M), S(M, R)\right\}
$$

(where $\lambda$ denote the number of periods $L$ has to wait to make an offer, if M's offer is rejected).

We give the formal proof in the appendix and sketch the intuition here. Notice that if the bargaining protocol is either $M L R$ or $R M L$, then $\mathrm{L}$ is not the formateur and $\lambda=1$.

If $\lambda=2$, then L's bargaining position is weak, which actually makes $\mathrm{L}$ an attractive coalition partner for M, and the LM coalition forms in equilibrium. To see this, suppose in order to derive a contradiction - that the MR coalition forms in equilibrium. Now if $\mathrm{M}$ should make an offer to $\mathrm{L}$, then if $\mathrm{L}$ rejects, $\mathrm{R}$ will make the next proposal (since $\lambda=2$ ); by stationarity, the MR coalition forms, and $\mathrm{L}$ is left out with a negative payoff $-x_{L}(M, R)$. Thus, sequential rationality forces L to accept M's offer, even if it gives L zero surplus. Since $S(L, R)>S(M, R)$, offering $\mathrm{L}$ zero surplus is sure to make $\mathrm{M}$ better off than a coalition with R. This contradiction shows that the LM coalition always forms when $\lambda=2$.

If instead $\lambda=1$, then the MR coalition can form if the difference between $S(L, M)$ and $S(M, R)$ is small enough. When $\lambda=1$, L can more easily reject a proposal from $\mathrm{M}$ than when $\lambda=2$, because $\lambda=1$ means $\mathrm{L}$ can immediately counter-offer (without $\mathrm{R}$ intervening). In this sense, L's bargaining position vis-a-vis $\mathrm{M}$ is strong when $\lambda=1$. Conversely, $\mathrm{R}$ is willing to accept any offer, even one that gives it zero surplus. Indeed, if $\mathrm{R}$ rejects M's offer, then $\mathrm{L}$ will make the next proposal and $\mathrm{R}$ will be left out (with a negative payoff $\left.-x_{R}(L, M)\right)$. In this situation, $\mathrm{M}$ prefers to make an offer to $\mathrm{R}$ if $S(M, R)$ is not too small. In a sense, L's bargaining "power" is actually a handicap, unless either $\mathrm{L}$ is the formateur and so can preempt all other proposals, or $S(M, R)$ is small enough to make $\mathrm{R}$ irrelevant.

\subsection{Random recognition protocol}

We now characterize the SSPE for the random recognition protocol. Here stationarity means behavior is independent of what happened in past periods, i.e. history independence in the usual sense. In each period, recognition probabilities are given by the seat shares $n(L), n(M)$ and $n(R)$ for $L, M$ and $R$ respectively. The SSPE is, in general, not in pure strategies. The mixing is between acceptance and rejection (unlike, for example, Ray, 2008). However, as $\delta \rightarrow 1$, the mixing becomes degenerate and the two closest parties, $\mathrm{L}$ and $\mathrm{M}$, form a government.

The formal analysis is relegated to the appendix, but we sketch the intuition here, retaining the notation $\phi_{P} \equiv n(P)$ as the recognition probability. We are primarily interested 
in equilibrium payoffs when $\delta$ is close to 1 . Fix an equilibrium, and let $\widetilde{s}_{P}$ denote the equilibrium continuation payoff of player $P \in\{L, M, R\}$. By definition, this is the payoff player $P$ expects to get in period $t+1$, if the period $t$ offer is rejected. Since we are considering stationary strategies, this does not depend on $t$, on who made the offer or rejected the offer, or any other aspect of past behavior. Note that if $P \neq M$ then $\widetilde{s}_{P}<0$ is possible, since player $P$ might expect to be left out of the government and suffer an externality. But $\widetilde{s}_{M}>0$ always holds, since a coalition government between $L$ and $R$ is ruled out. The minimum amount player $P$ can get if he is part of a coalition government is 0 . Let $s_{P} \equiv \max \left\{\widetilde{s}_{P}, 0\right\}$.

Consider player $L$. Suppose, in order to derive a contradiction, that $s_{L}=0$. Then $M$, in any period where he is recognized to make a proposal, will certainly propose that he and $L$ form a government where $M$ gets all the surplus $S(L, M)$ and $L$ gets 0 . (This is accepted because if $L$ rejects he expects $\delta \widetilde{s}_{L} \leq \delta s_{L}=0$ anyway.) If instead $L$ is recognized, he will certainly propose that he and $M$ form a government where $M$ gets $\delta s_{M}$ (which makes $M$ indifferent between accepting and rejecting). What happens if $R$ is recognized ? Consider two alternatives: $M$ never finds any offer from $R$ attractive, or $M$ accepts an offer from $R$. If the former is true, then whenever $R$ makes a proposal, it is rejected and the game progresses to the next round. Then $M$ gets $\delta s_{M}$ whether $L$ makes an offer to $M$ which he accepts, or $R$ makes a proposal which is rejected, so we have,

$$
s_{M}=\left(\phi_{L}+\phi_{R}\right) \delta s_{M}+\phi_{M} S(L, M)
$$

Since $\phi_{L}+\phi_{M}+\phi_{R}=1, s_{M} \rightarrow S(L, M)$ as $\delta \rightarrow 1$. This means $R$ cannot make an acceptable offer to $M$, since $S(M, R)<S(L, M)$. However, $L$ will then never suffer the negative externality from not being in government, so

$$
\widetilde{s}_{L} \geq \phi_{L}\left(S(L, M)-\delta s_{M}\right)>0
$$

Therefore, $s_{L}$ cannot be 0 , contradicting our hypothesis. So $M$ must get an acceptable offer from $R$ in equilibrium. Now $s_{L}$ might be zero if the negative externality offsets the small positive expected benefit from the $L M$ coalition to $L$. But the value of $s_{M}$ is unaffected and is close to $S(L, M)$ for high $\delta$, so $R$ cannot in fact make an acceptable offer to $M$. Therefore, there is no externality on $L$, and again $s_{L}$ cannot be 0 . This contradiction shows that we cannot have a pure strategy SSPE in which $s_{L}=0$.

It must therefore be true that $s_{L}=\widetilde{s}_{L}>0$. Again, suppose $R$ cannot make an acceptable offer to $M$. Now, essentially, the bargaining is between $L$ and $M$, with no agreement reached in periods where $R$ is recognized. "Bargaining power" in this bilateral bargaining is directly related to the recognition probabilities. Thus, $s_{M} \rightarrow \frac{\phi_{M}}{\phi_{M}+\phi_{L}} S(L, M)$ as $\delta \rightarrow 1$. If 
$S(M, R)<\frac{\phi_{M}}{\phi_{M}+\phi_{L}} S(L, M)$ then this indeed gives us an equilibrium. However, if $S(M, R)>$ $\frac{\phi_{M}}{\phi_{M}+\phi_{L}} S(L, M)$, then there is enough surplus in the $M R$ coalition that $R$ could intervene with an acceptable offer to $M$, contradicting our hypothesis.

What does the equilibrium look like if $S(M, R)>\frac{\phi_{M}}{\phi_{M}+\phi_{L}} S(L, M)$ ? Now $R$ must be able to make an acceptable offer to $M$. Can we have $s_{L}>0$ in a pure strategy equilibrium? Once again, $M$ is the only player in every agreement and as $\delta \rightarrow 1$, his loss from not being recognized becomes lower and lower, as does $L$ 's payoff when recognized. Now the negative payoff $L$ will get if $R$ is recognized makes $L$ willing to accept 0 in a coalition with $M$, but then $s_{L}>0$ is impossible. Thus, a pure strategy SSPE does not exist in this case, since we have ruled out both $s_{L}=0$ and $s_{L}>0$. We must allow randomization in equilibrium. ${ }^{9}$

In general, randomization can either be in choice of partners as a proposer, or in deciding to accept or reject as a responder. Consider the first possibility. It follows directly from our previous discussion that this is impossible; $M$ is the only one who can randomize (since the other two each can choose only $M$ ) and any randomization by $M$ as proposer will drive $L$ 's expected payoff even lower (in our earlier discussion, $M$ was offering to $L$ with probability 1). Therefore the only possible stationary equilibrium must have $M$ randomizing between accepting and rejecting offers. Clearly, this cannot apply to offers from $L$, because $\delta s_{M}<S(L, M)$ in equilibrium, and $L$ can force $M$ to accept with probability 1 by offering $\varepsilon>0$ more than $\delta s_{M}$. Therefore, $M$ must instead randomize in accepting or rejecting $R$ 's offer. This also determines the offer by $R$, which must be $S(M, R)$ (so $R$ cannot force $M$ to accept with probability 1 by raising the offer). It turns out (to maintain $s_{L}>0$ ) that the offer is in fact accepted with a probability that goes to 0 as $\delta \rightarrow 1$, so $R$ essentially never participates in government, although his presence at the bargaining table influences the way $L$ and $M$ split the surplus. Thus, we get the following result.

Proposition 2 For $\delta$ close to 1, the bargaining game with random recognition has a unique SSPE outcome. As $\delta \rightarrow 1$ the LM coalition always forms and M's share of the surplus converges to

$$
s_{M}=\max \left\{\frac{n(M)}{n(L)+n(M)} S(L, M), S(M, R)\right\}
$$

\footnotetext{
${ }^{9}$ To see the intuition behind the non-existence, consider a simpler case without policy preferences, where any coalition government would generate the same surplus and there would be no externalities. The only heterogeneity would come from the $\phi_{P}$. Suppose in this case the equilibrium payoffs are ordered in the same way as $\phi_{P}$, and suppose this order is $L, M, R$. If $M$ has a strictly higher continuation payoff than $R$, then both $L$ and $M$, as proposers, will choose $R$, who will be in every coalition and will therefore have very high payoff, contradicting the supposed equilibrium configuration. To avoid the contradiction, there must be randomisation in equilibrium to ensure that at least two of the players have the same equilibrium payoff.
} 
The formal proof is in the Appendix.

\section{Incentives to form ex ante coalitions with Propor- tional Representation}

A coalition formed before the election is called an ex ante coalition. Propositions 1 and 2 establish the outcome in the absence of any ex ante coalitions. (We assume $\delta$ is close enough to 1 to make it legitimate to consider the limit as $\delta \rightarrow 1$.) We say that an ex ante agreement is viable if both coalition partners are made strictly better off by signing the ex ante agreement, compared to the outcome with no ex ante coalitions. In this section (and in section 5) we consider whether viable ex ante coalitions exist. In other words, does some point in the utility-possibility set for a two-party ex ante coalition give both parties higher payoff than no ex ante agreement? If so, then presumably an ex ante coalition will form, although its precise form depends on ex ante bargaining strength. For example, consider the pre-election bargaining game of Morelli (2004), where M makes a take-it-or-leave-it offer to a party of its choice. In this case, if a viable ex ante agreement exists, then M's optimal offer is the viable ex ante agreement which gives M the highest payoff, and this offer must be accepted in equilibrium. Conversely, if no viable ex ante agreement exists, then no ex ante coalition forms; because any agreement that makes $\mathrm{M}$ better off must make the coalition partner worse off (than no agreement), hence it must be rejected in equilibrium. Thus, with the Morelli (2004) ex ante bargaining game, an ex ante agreement is signed if and only if a viable ex ante coalition exists. However, rather than focus on this particular ex ante bargaining game, we will map out the set of viable ex ante coalitions.

In this section, we consider proportional representation (PR). An ex ante coalition $\left\{P, P^{\prime}\right\}$ is a joint national list. If there were no ex ante agreement, party P's vote share would be $n(P)=v(P)$. We need to define "sincere voting" for the case of a joint list. We will assume all $P$ supporters and all $P^{\prime}$ supporters vote for the joint $\left\{P, P^{\prime}\right\}$ list, hence the list gets $v(P)+v\left(P^{\prime}\right)$ seats in the election. The ex ante agreement allocates these seats among the two parties, by specifying how many (and in which order) candidates from each party appear on the joint list. (If candidates from $P$ and $P^{\prime}$ alternate on the joint list, then each party gets half of the $v(P)+v\left(P^{\prime}\right)$ seats, but unequal divisions are attainable by putting more candidates from one party on the list, or putting them higher up.) Again, there is nothing else on the table ex ante.

The ex ante agreement divides up seat shares within the coalition, but these seats are translated into payoffs in a non-linear way, via the recognition rule. Moreover, there is 
a discontinuity when a party's seat share surpasses 0.5 , since the majority party forms a one-party government and gets all the rents from office. This partial non-transferability of utility, and the discontinuity at 0.5 , leads to an ex ante coalition-formation problem which is somewhat non-standard.

\subsection{ASB bargaining ex post}

There are two cases to consider.

Case 1: In the absence of ex ante agreement, $L$ and $M$ would form a coalition government ex post.

Proposition 1 gives the conditions under which case 1 occurs. In this case, it is impossible that $\mathrm{L}$ and $\mathrm{M}$ have a viable ex ante coalition. Indeed, $\mathrm{L}$ and $\mathrm{M}$ cannot increase their total number of seats by a joint list under PR, and (by definition of case 1) they would form a government even with no ex ante agreement, so both parties cannot be strictly better off with a joint list. An ex ante coalition between $\mathrm{M}$ and $\mathrm{R}$, however, might be viable. Their joint list would win

$$
n(M)+n(R)=v(M)+v(R)
$$

seats. There are three ways the joint MR list could be viable, which we discuss in turn.

(i) If the MR list results in M getting its own majority in parliament $(n(M) \geq 1 / 2)$, then the government will be an M-party majority government (rather than an LM coalition), and this could benefit both $\mathrm{M}$ and $\mathrm{R}$. Now $n(M) \geq 1 / 2$ implies $n(R) \leq v(M)+v(R)-1 / 2$. That is, to achieve an M-party majority, $\mathrm{R}$ must give up at least $\frac{1}{2}-v(M)$ seats to $\mathrm{M}$. This certainly makes $\mathrm{M}$ better off, and $\mathrm{R}$ gains $x_{R}(L M)-x_{R}(M)$ by blocking $\mathrm{L}$ from joining the government. The MR ex ante coalition is viable if $\mathrm{R}$ can be made better off, i.e., if

$$
x_{R}(L M)-x_{R}(M)>\alpha\left(\frac{1}{2}-v(M)\right) .
$$

This condition requires that a coalition government involving L imposes a significant negative externality on R.

(ii) The ex ante coalition between $\mathrm{M}$ and $\mathrm{R}$ could change seat shares, and thus the ex post bargaining protocol, in such a way that the coalition government becomes MR rather than LM. By Proposition 1, this can only happen if $\mathrm{M}$ and $\mathrm{R}$ are not too ideologically distant,

i.e., if $S(M, R)>\frac{1}{3} S(L, M)$. If this inequality holds, then the MR coalition forms ex post if $\mathrm{L}$ is not the formateur and $\lambda=1$. To accomplish this, $\mathrm{R}$ transfers sets to $\mathrm{M}$ via the joint list. This transfer would, on the one hand, benefit $M$ by increasing his seat share, thus giving him a motive to sign the ex ante agreement. Simultaneously, by "shrinking", R makes himself a 
more attractive coalition partner ex post Specifically, if $v(M)>v(R)>v(L)$, then with no ex ante agreement the protocol is $M R L$, and Proposition 1 implies that the LM government would form ex post. But if, by forming a joint list, $\mathrm{R}$ transfers $v(R)-v(L)$ seats to $\mathrm{M}$, then $\mathrm{R}$ becomes the smallest party and the ex post bargaining protocol $M L R$. Note that $\lambda$ changes from 2 to 1 and, by Proposition 1, the ex post government changes to MR. Party R loses $v(R)-v(L)$ seats but now will be part of the coalition government, receiving a share $S(M, R)-s^{1}(M)$ of the ex post surplus, and avoiding the externality $x_{R}(L M)$. Party M's share of the surplus falls from $s^{2}(M)$ to $s^{1}(M)$, but as compensation he gains $v(R)-v(L)$ seats. Both $\mathrm{M}$ and $\mathrm{R}$ are made better off if

$$
S(M, R)-s^{1}(M)+x_{R}(L M)>\alpha(v(R)-v(L))>s^{2}(M)-s^{1}(M)
$$

But there are other possibilities. If $v(L)>v(M)>v(R)$ then the bargaining protocol without ex ante agreements is $L M R$. If by forming a joint list, $\mathrm{R}$ transfers $v(L)-v(M)$ seats to $\mathrm{M}$, then the ex post bargaining protocol becomes $M L R$. Again $\lambda$ changes from 2 to 1 and the ex post government changes from LM to MR. Both M and R are made better off if

$$
S(M, R)-s^{1}(M)+x_{R}(L M)>\alpha(v(L)-v(M))>s^{2}(M)-s^{1}(M)
$$

If instead $v(L)>v(R)>v(M)$ then the bargaining protocol without ex ante agreements is $L R M$. If by forming a joint list, $\mathrm{R}$ transfers $v(L)-v(M)$ seats to $\mathrm{M}$, then the ex post bargaining protocol becomes $M L R$. Here $\lambda$ remains 1 but $\mathrm{L}$ is no longer the formateur, and by Proposition 1 the ex post government changes from LM to MR. Party M is certainly better off because he gets more seats while his share of the surplus remains $s^{1}(M)$, and party $\mathrm{R}$ is better off if

$$
S(M, R)-s^{1}(M)+x_{R}(L M)>\alpha(v(R)-v(L))
$$

(iii) The MR ex ante coalition might change the bargaining power within the LM government in M's favor. If $\lambda=1$, then $\mathrm{R}$ and $\mathrm{M}$ could both gain from an ex ante agreement where $\mathrm{M}$ transfers seats to $\mathrm{R}$, so that $\lambda$ changes from 1 to 2 . For example, if $v(L)>v(R)>v(M)$, then with no ex ante agreement the protocol is LRM with $\lambda=1$; but if $M$ transfers $v(L)-v(R)$ seats to $\mathrm{R}$, then $\mathrm{R}$ becomes the biggest party ex post and $\mathrm{M}$ remains the smallest, hence the ex post protocol is RLM, with $\lambda=2$. This transfer of seats certainly makes R strictly better off, and M's share of the ex post surplus increases from $s^{1}(M)$ to $s^{2}(M)$ (as defined in (2)) due to his increased bargaining power vis-a-vis L. Thus, M is strictly better off if

$$
\lambda=1 \quad \text { and } \quad s^{2}(M)-s^{1}(M)>\alpha \Delta
$$


where $\Delta$ denotes the minimum number of seats that M needs to transfer to $\mathrm{R}$ to change $\lambda$ from 1 to 2. For example, if $v(L)>v(R)>v(M)$ then $\Delta=v(L)-v(R)$.

We summarize this discussion:

Proposition 3 Assume $P R$ and $A S B$ bargaining ex post. In case 1, $L$ and $M$ do not have a viable ex ante coalition. An ex ante coalition between $M$ and $R$ could be viable in several ways: if (3) holds; if $v(M)>v(R)>v(L)$ and (4) holds; if $v(L)>v(M)>v(R)$ and (5) holds; if $v(L)>v(R)>v(M)$ and (6) holds; or if (7) holds.

Since M prefers to get its own majority, we can characterize the equilibrium when the Morelli (2004) bargaining game is played ex ante.

Corollary 1 Assume PR and ASB bargaining ex post. Suppose case 1 applies and M makes a take-it-or-leave-it offer ex ante. If (3) holds, then the $M R$ ex ante coalition forms, and $M$ forms a majority government ex post. Otherwise, the outcome may be an $M R$ ex ante coalition but either an LM or MR coalition government ex post, depending on which of the conditions listed in Proposition 3 holds.

Case 2: In the absence of ex ante agreement, $M$ and $R$ would form a coalition government ex post.

Proposition 1 gives the conditions under which case 2 occurs: $S(M, R)>\frac{1}{3} S(L, M)$ and the bargaining protocol (in the absence of ex ante agreements) is either $M L R$ or $R M L$. Here, $\mathrm{M}$ and $\mathrm{R}$ cannot have a viable ex ante coalition (for the same reason that LM could not be viable in case 1 ). However, the ex ante coalition between $\mathrm{L}$ and $\mathrm{M}$ might be viable for several reasons. First, it might allow $\mathrm{M}$ to form a majority government. Such an ex ante agreement is viable if a coalition government which includes $\mathrm{R}$ has a big negative externality on L. The condition analogous to $(3)$ is

$$
x_{L}(M R)-x_{L}(M)>\alpha\left(\frac{1}{2}-v(M)\right)
$$

The second way the ex ante coalition between L and M could be viable is if the ex ante agreement affects seat shares in such a way that the ex post bargaining protocol changes and the coalition government becomes LM rather than MR. This can be achieved in two ways: either by transferring seats from $\mathrm{M}$ to $\mathrm{L}$, or by transferring seats from $\mathrm{L}$ to $\mathrm{M}$. For example, if the bargaining protocol without ex ante agreements would be $M L R$, then $\mathrm{L}$ can transfer $v(L)-v(R)$ seat shares to $\mathrm{M}$, making the new bargaining protocol $M R L$. Party $\mathrm{M}$ certainly gains from this. Party L loses seat shares, but now will be part of the coalition 
government, receiving a share $S(L, M)-s^{2}(M)$ of the ex post surplus, and avoiding the externality $x_{L}(M R)$. L is better off if

$$
S(L, M)-s^{2}(M)+x_{L}(M R)>\alpha(v(M)-v(L))
$$

But another way to change the coalition government from MR to LM is for $\mathrm{M}$ to transfer $(v(M)-v(L)) / 2$ seats to $L$, making the new bargaining protocol $L M R$ (instead of $M L R$ ) Party L certainly gains from this. Party M loses seat shares, but gets a bigger share of the ex post surplus because $\lambda$ has changed from 1 to 2 . M is better off if

$$
s^{2}(M)-s^{1}(M)>\alpha \frac{v(M)-v(L)}{2}
$$

Analogous arguments can be made if the bargaining protocol without ex ante agreements would be $R M L$. In either case, let $\Delta^{\prime}$ denote the number of seats that $\mathrm{L}$ must transfer to $\mathrm{M}$ in order to change the coalition government from MR to LM, and let $\Delta^{\prime \prime}$ denote the number of seats that M must transfer to L in order to achieve the same outcome. Then we get the following two conditions, corresponding to (9) and (10):

$$
\begin{gathered}
S(L, M)-s^{2}(M)+x_{L}(M R)>\alpha \Delta^{\prime} \\
s^{2}(M)-s^{1}(M)>\alpha \Delta^{\prime \prime}
\end{gathered}
$$

We can summarize as follows.

Proposition 4 Assume $P R$ and $A S B$ bargaining ex post. In case 2, $M$ and $R$ do not have a viable ex ante coalition, but an ex ante coalition between $M$ and $L$ is viable if either (8), (11) or (12) holds.

As before, we have a corollary.

Corollary 2 Assume PR and ASB bargaining ex post. Suppose case 2 applies, and $M$ makes a take-it-or-leave-it offer ex ante. If (8) holds, then the $M R$ ex ante coalition forms, and $M$ forms a majority government ex post. If (8) is violated but either (11) or (12) holds, then the LM coalition forms both ex ante and ex post.

\subsection{Random recognition ex post}

With the ASB protocol, as discussed in section 4.1, M can give up seats in an ex ante agreement in a way which changes the ex post protocol in his own favor (changes $\lambda$ from 
1 to 2). In contrast, with random recognition the only way $M$ can increase his ex post bargaining power is by increasing his own seat share $n(M)$ (see Proposition 2). But the other parties would never agree to transfer seats to M just to increase M's ex post bargaining power. (There are no "side payments" ex ante which M can use to "buy" seats; seats are the only currency). Thus, under random recognition an ex ante coalition cannot form simply to manipulate the bargaining power within a given government. It could, however, produce a majority government. Proposition 2 implies that if no party has its own majority then R's payoff is $\alpha v(R)-x_{R}(L, M)$. If, however, $\mathrm{M}$ and $\mathrm{R}$ form an ex ante coalition wherein $\mathrm{R}$ gives up $\frac{1}{2}-v(M)$ seats to $\mathrm{M}$, then $\mathrm{M}$ gets its own majority and R's payoff is $\alpha v(R)-\alpha\left(x_{R}(L M)-x_{R}(M)\right)-x_{R}(M)$. Clearly, this agreement benefits $\mathrm{M}$, and it benefits $\mathrm{R}$ as long as

$$
x_{R}(L M)-x_{R}(M)>\alpha\left(\frac{1}{2}-v(M)\right)
$$

Thus, an MR ex ante coalition might form in order to reduce the externality on $\mathrm{R}$ and give M a majority. The LM ex ante coalition cannot possibly be viable. Thus, we get

Proposition 5 Assume $P R$ and random recognition bargaining ex post. $M R$ is a viable ex ante coalition if and only if (13) holds. The $M R$ ex ante coalition, if it forms, always leads to an $M$ majority government. No other ex ante coalition can be viable.

We have the following corollary:

Corollary 3 Assume $P R$ and random recognition bargaining ex post. Suppose $M$ makes a take-it-or-leave-it offer ex ante. If (13) holds the MR coalition forms ex ante, but after the election $M$ forms a one-party government. If (13) is violated then there is no ex ante coalition.

\section{Incentives to form ex ante coalitions with Plurality Voting}

In the case of plurality voting, an ex ante agreement divides up the districts, and specifies in which districts each party should run and where it should drop out of the race. This is the only item on the table for ex ante negotiations. In particular, an ex ante coalition cannot make commitments about behavior in the post-election stage. With no ex ante agreement, party P's vote share would be $w(P)$. Our "sincere voting" assumption implies that if a party drops out of a district, its supporters will vote for the ideologically closest party. Thus, if $\mathrm{M}$ drops out of a district, the M-supporters in the district vote for $\mathrm{L}$; if $\mathrm{L}$ drops out the 
L-supporters vote for $\mathrm{M}$. In either case, $\mathrm{R}$ only wins the district if his supporters form a majority. Because of this, if $\mathrm{L}$ and $\mathrm{M}$ form an ex ante coalition, they can reduce R's seat share from $w(R)$ to $z(R)$. The remaining $w(R)-z(R)>0$ seats can be redistributed within the LM coalition (by dividing up the districts appropriately) to make both L and M better off. In other words, $\mathrm{L}$ and $\mathrm{M}$ can always benefit by not "splitting the vote". This is true whether ex post bargaining is random recognition or ASB. Thus, we get:

Proposition 6 Under PV, the LM ex ante coalition is always viable.

However, there are other reasons to form ex ante coalitions, including the MR coalition. But notice that there is an asymmetry in the way parties can transfer seats. $\mathrm{R}$ can transfer seats to $\mathrm{M}$ (at the rate $1-z(L)$ ) because if $\mathrm{R}$ drops out of a district, then the $\mathrm{R}$-supporters vote for $\mathrm{M}$, and $\mathrm{M}$ wins the district unless the L-supporters form a majority. But $\mathrm{M}$ cannot transfer any seats to $\mathrm{R}$, because if $\mathrm{M}$ drops out of a district, the M-supporters vote for $\mathrm{L}$ (since, ideologically, $\mathrm{M}$ is closer to $\mathrm{L}$ than to $\mathrm{R}$ ). Despite this non-transferability, it is possible for the MR coalition to form ex ante. To study this, we need to be more specific about the ex post bargaining protocol.

\subsection{ASB bargaining ex post}

Apart from not splitting the vote, there are two other possible motives for an ex post coalition: to influence which government will form, and to manipulate the bargaining power within the government. It is useful to distinguish two cases, depending on what would happen ex post in the absence of ex ante agreements.

Case 1: In the absence of ex ante agreement, $\mathrm{L}$ and $\mathrm{M}$ would form a coalition government. (The conditions for this to happen are given in Proposition 1.)

We distinguish two sub-cases.

Sub-case 1a: $\mathrm{R}$ is ex post irrelevant in the sense that $S(M R)<\frac{1}{3} S(L M)$.

For the MR coalition to be viable ex ante, $\mathrm{R}$ must be made better off than if there is no ex ante coalition. By Proposition 1, in case 1a, $\mathrm{R}$ will never be part of a coalition government. Moreover, recall that $\mathrm{M}$ cannot transfer seats to $\mathrm{R}$ under PV. The only way the ex ante agreement can make $\mathrm{R}$ better off is if it leads to a majority government formed by $\mathrm{M}$ (rather than an LM coalition government). For this to happen, $\mathrm{R}$ must drop out of some districts in order to raise M's seat share to $1 / 2$. If $\mathrm{R}$ drops out of $y$ districts, then $\mathrm{M}$ will win a fraction $1-z(L)$ of these, and a fraction $w(M)$ of the remaining $1-y$ districts. Thus, $y$ must satisfy

$$
(1-y) w(M)+y(1-z(L)) \geq \frac{1}{2}
$$


The smallest such $y$ is

$$
y=\frac{\frac{1}{2}-w(M)}{1-z(L)-w(M)}
$$

The cost to $\mathrm{R}$ of dropping out of $y$ districts is $\alpha w(R) y$ (since he would win a fraction $w(R)$ in a three-way race). On the other hand, $\mathrm{R}$ gains $x_{R}(L M)-x_{R}(M)$ if $\mathrm{M}$ forms a one-party government rather than a coalition with L. Therefore, the condition for the MR ex ante coalition to be viable is that the negative externality a LM coalition government imposes on $\mathrm{R}$ is sufficiently big:

$$
x_{R}(L M)-x_{R}(M)>\alpha w(R) \frac{\frac{1}{2}-w(M)}{1-z(L)-w(M)}
$$

We thus have the following proposition:

Proposition 7 Assume PV and ASB bargaining ex post. In case 1a the MR ex ante coalition is viable if and only if (14) holds.

Thus, there can be multiple viable ex ante coalitions. To make a more precise prediction, suppose $\mathrm{M}$ makes a take it or leave it offer ex ante. Propositions 6 and 7 together imply that if (14) is violated, then LM forms ex ante (since no other viable ex ante coalition exists). However, if (14) holds then M has a choice between the LM and the MR coalitions - both are viable. The MR ex ante coalition would give $\mathrm{M}$ a majority of the seats. For M to prefer the LM ex ante coalition over the MR ex ante coalition, the LM coalition must give him even more seats.

In the MR coalition, the greatest number of seats that $\mathrm{M}$ can get will make $\mathrm{R}$ indifferent between accepting and rejecting M's take it or leave it offer. Suppose the offer specifies that $\mathrm{R}$ drops out of $y$ districts. Then, the indifference condition implies that $y$ satisfies

$$
\alpha w(R) y=x_{R}(L M)-x_{R}(M)
$$

Using (15) to substitute for $y$, the number of seats M gets is

$$
(1-y) w(M)+y(1-z(L))=w(M)+(1-z(L)-w(M)) \frac{x_{R}(L M)-x_{R}(M)}{\alpha w(R)}
$$

Consider the LM ex ante coalition. Suppose M drops out of $m$ districts, and L drops out of the remaining $1-m$ districts. In the $m$ districts, $\mathrm{L}$ gets the votes of both $\mathrm{L}$ and $\mathrm{M}$ supporters, so he wins unless the $\mathrm{R}$ supporters have a majority. Thus, L gets $m(1-z(R))$ 
seats. However, M must get a majority, otherwise he would not propose the LM coalition. Thus, L's payoff from joining the LM ex ante coalition would be $\alpha m(1-z(R))-x_{L}(M)$. On the other hand, if there is no ex ante coalition, then Proposition 1 implies that $\mathrm{L}$ gets

$$
\alpha w(L)+\frac{3-\lambda}{3} S(L, M)
$$

(where $\lambda$ is defined as in Proposition 1 ). The smallest feasible $m$ will make $\mathrm{L}$ indifferent between accepting and rejecting M's offer:

$$
\alpha m(1-z(R))-x_{L}(M)=\alpha w(L)+\frac{3-\lambda}{3} S(L, M)
$$

In the $1-m$ districts, $\mathrm{M}$ wins unless the $\mathrm{R}$ supporters have a majority. Thus, using (17) to substitute for $m$, the greatest number of seats $M$ can get in a coalition with $\mathrm{L}$ is

$$
(1-m)(1-z(R))=1-z(R)-w(L)-\frac{1}{\alpha}\left(\frac{3-\lambda}{3} S(L, M)+x_{L}(M)\right)
$$

M prefers the LM coalition if and only if (18) exceeds (16), which is true if and only if

$$
\alpha(w(R)-z(R))>(1-z(L)-w(M)) \frac{x_{R}(L M)-x_{R}(M)}{w(R)}+\frac{3-\lambda}{3} S(L, M)+x_{L}(M)
$$

Since $w(R)>z(R)$, this inequality holds if seats are sufficiently valuable (i.e., $\alpha$ is big). This is intuitive. Recall that if (14) holds then $\mathrm{M}$ always has the option to make an offer to $\mathrm{R}$ ex ante, this will be accepted, and $\mathrm{R}$ drops out of sufficiently many districts to allow $\mathrm{M}$ to form a one-party government. $\mathrm{R}$ is willing to do this to eliminate the negative externality created by the LM government. The more important is the externality, or - for a given externality - the less valuable are seats, the more districts $\mathrm{R}$ is willing to drop out of. On the other hand, the LM coalition avoids "splitting the vote" - it reduces R's seat share from $w(R)$ to $z(R)$. The extra seats can be allocated between $\mathrm{M}$ and L. Even if, as a result of the ex ante coalition, $\mathrm{M}$ gets a majority of seats and forms a majority government ex post, L may still be willing to participate if seats are sufficiently valuable. From this it follows intuitively that if seats are sufficiently valuable (specifically, (19) holds) then the LM coalition will form. Otherwise, MR forms.

To summarize, we have shown:

Proposition 8 Assume $P V$ and $A S B$ bargaining ex post. Suppose case 1a applies, and $M$ makes a take it or leave it offer ex ante. (i) If (14) holds then both LM and MR are viable ex ante coalitions; LM forms in equilibrium if (19) holds, otherwise MR forms. (In either case, 
$M$ will eventually form a one-party government). (ii) If (14) is violated then LM forms ex ante, being the only viable ex ante coalition.

Sub-case 1b: $\mathrm{R}$ is ex post relevant in the sense that $S(M R)>\frac{1}{3} S(L M)$.

In this case, both MR and LM ex ante coalitions are again possible, depending on the parameters. As in case 1a, the MR coalition tends to form when $\alpha$ is low, and LM forms otherwise. A more detailed consideration is relegated to the appendix. Note that the agreements can be motivated by trying to avoid splitting the votes or by trying to change the bargaining power of parties ex post. Incomplete seat sharing arrangements are also possible.

Finally, we consider the simplest case.

Case 2: In the absence of ex ante agreement, $M$ and $R$ would form a coalition government ex post.

By Proposition 1, this is the case when $S(M, R)>\frac{1}{3} S(L, M)$ and the bargaining protocol in the absence of ex ante agreements would be either $M L R$ or $R M L$. In this case, there is no viable ex ante coalition between $\mathrm{M}$ and $\mathrm{R}$. (Recall that $\mathrm{R}$ cannot increase its seats share by any arrangement with M). Thus, from Proposition 6, we have:

Proposition 9 Assume $P V$ and $A S B$ bargaining ex post. In case 2, the unique viable ex ante coalition is $L M$.

\subsection{Random recognition ex post}

According to Proposition 2, with random recognition player R is always "ex post irrelevant" in the sense that he cannot possibly be part of a coalition government. The analysis is, therefore, similar to Case 1a of Section 5.1. The MR ex ante coalition is viable only if the negative externality a LM coalition government imposes on $\mathrm{R}$ is sufficiently big, which is true if and only if (14) holds. We thus have the following result:

Proposition 10 Assume $P V$ and random recognition ex post. The MR ex ante coalition is viable if and only if (14) holds.

To make a more precise prediction, we again suppose $\mathrm{M}$ makes a take it or leave it offer ex ante. If (14) is violated then, of course, LM forms (in order to avoid splitting the vote). But if (14) holds, both LM and MR are viable ex post, and M will propose the coalition that gives him a higher payoff.

In the MR coalition, the greatest number of seats that $M$ can get is again given by (16). Consider the LM ex ante coalition which, again, has to lead to a one-party M government 
for $\mathrm{M}$ to be interested. If $\mathrm{M}$ drops out of $m$ districts, and $\mathrm{L}$ drops out of the remaining $1-m$ districts then, as before, L's payoff would be $\alpha m(1-z(R))-x_{L}(M)$. On the other hand, if there is no ex ante coalition, then Proposition 2 implies that $\mathrm{L}$ gets $\alpha w(L)+K$, where

$$
K \equiv \min \left\{\frac{w(L)}{w(L)+w(M)} S(L, M), S(L, M)-S(M, R)\right\}
$$

The smallest feasible $m$ will make L indifferent between accepting and rejecting M's offer:

$$
\alpha m(1-z(R))-x_{L}(M)=\alpha w(L)+K
$$

In the $1-m$ districts, $\mathrm{M}$ wins unless the $\mathrm{R}$ supporters have a majority. Thus, using (17) to substitute for $m$, the greatest number of seats $\mathrm{M}$ can get in a coalition with $\mathrm{L}$ is

$$
(1-m)(1-z(R))=1-z(R)-w(L)-\frac{x_{L}(M)+K}{\alpha}
$$

M prefers the LM coalition if and only if (21) exceeds (16), which is true if and only if

$$
\alpha(w(R)-z(R))>(1-z(L)-w(M)) \frac{x_{R}(L M)-x_{R}(M)}{w(R)}+x_{L}(M)+K
$$

The situation is, therefore, again as in Case 1a of Section 5.1. Since $w(R)>z(R)$, the inequality holds if seats are sufficiently valuable ( $\alpha$ big), and the intuition is as before.

To summarize, we have shown:

Proposition 11 Assume $P V$ and random recognition bargaining ex post. Suppose $M$ makes a take it or leave it offer ex ante. (i) If (14) holds then both LM and MR are viable ex ante coalitions; LM forms in equilibrium if (22) holds, otherwise MR forms. (In either case, $M$ will eventually form a one-party government). (ii) If (14) is violated then LM forms ex ante, being the only viable ex ante coalition.

\section{Strategic Voting}

So far, we have considered a game of sincere voting, where voters always vote for the candidate who is the closest in terms of ideological position, while the "parties" manipulate the outcome of the elections by forming ex ante coalitions. We now briefly discuss whether these ex ante manipulations can be replicated by strategic voting, and find the answer is "no". Morelli (2004) studies a similar issue, and Indridason (2009) studies how the expected ex post coalitions in PR affect strategic voter behavior. 
To stay close to the previous framework, we assume all P-supporters have the same payoff function as party $\mathrm{P} .{ }^{10}$ The ex ante coalition formation stage is replaced by an ex ante "recommendation stage", where each party tells its supporters how to vote in the upcoming election. Since all P-supporters have common interests, they have no reason to disobey the instructions coming from party $\mathrm{P}$. In the recommendation stage, players $\mathrm{L}, \mathrm{M}$ and $\mathrm{R}$ make their recommendations simultaneously. In the proportional representation electoral system, the recommendation of player $\mathrm{P}$ allocates its $v(P)$ supporters (and hence $v(P)$ seats) among the three parties. In the case of PV, a recommendation advises supporters in each district whom they should vote for. After the recommendation stage, the election is held, followed by post-election bargaining as before. We call this three-stage game the strategic voting game.

In the strategic voting game, the parties can transfer seats in ways that would be impossible to achieve by ex ante coalitions and sincere voting. For example, with sincere voting it was impossible for $M$ to transfer seats to $R$, and as we saw above, this influenced whether the MR coalition could be viable ex ante. In contrast, with strategic voting $\mathrm{M}$ can transfer seats to $\mathrm{R}$ by simply recommending some M-supporters to vote for $\mathrm{R}$. With plurality voting, $\mathrm{M}$ and $\mathrm{R}$ could make an implicit agreement: $\mathrm{M}$ recommends that in some districts, the $\mathrm{M}-$ supporters vote for $\mathrm{R}$, while $\mathrm{R}$ recommends that in the remaining districts the $\mathrm{R}$-supporters vote for $\mathrm{M}$. This would reduce L's vote share to $z(L)$, and both $\mathrm{R}$ and $\mathrm{M}$ could be made better off by this implicit agreement. However, such an implicit agreement, involving a twoway transfer of votes, could not be part of an equilibrium of the strategic voting game. Both $\mathrm{M}$ and $\mathrm{R}$ would have an incentive to violate the agreement at the recommendation stage by recommending that their voters should, in fact, vote for their own party. In effect, the strategic voting game lacks even the minimal commitment that ex ante coalitions allow in the sincere voting game.

Section 5 showed that $\mathrm{M}$ and $\mathrm{L}$ sometimes find it optimal to agree to two-way transfers of votes ex ante, in order to drive the vote share of $\mathrm{R}$ down to $z(R)$. However, for the reason explained in the previous paragraph, this cannot happen in equilibrium of the strategic voting game without binding commitments. (Both L and M would have an incentive to ask their voters to vote for their own party rather than voluntarily giving up seats.) In general, an equilibrium ex ante coalition that involves both parties withdrawing from some seats cannot be replicated by equilibrium recommendations in the strategic voting game. Due to lack of commitment, strategic voting cannot replicate the outcome of the sincere voting game.

\footnotetext{
${ }^{10}$ Thus, the P-supporters care about party P's number of seats in parliament, its share of the surplus from being in government, and the negative externality from ideologically distant parties in government. Intuitively, if a party is in government it can reward its base to an extent proportional to the amount of power it exerts. If the party concerned is not in government, its voters get no benefit and incur a loss corresponding to an undesirable policy.
} 
We have only discussed two-way transfers of votes. But since there is a larger feasible set of transfers under strategic voting, we cannot even be sure that ex ante coalitions involving one-way transfers can be replicated in equilibrium of the strategic voting game. Some possible transfers that were not possible with sincere voting might now be best responses. Also, for example, in the case of ASB with $\lambda=1, \mathrm{M}$ might want to give up seats to $\mathrm{R}$ to change $\lambda$ to 2. However, it is not necessarily a best response here for $\mathrm{L}$ to do nothing; it might ask its voters in some districts to vote for $\mathrm{M}$ to bring $\lambda$ back to 1 . Of course, it is not a best response for $M$ to give up vote share or seats if $L$ is going to counteract it, so this too cannot be an equilibrium. Equilibrium of the strategic voting game might require mixed strategies.

\section{Conclusion}

In this paper, we have considered the effect of post-election bargaining on ex ante seat-sharing arrangements. A key assumption was very limited commitment power ex ante. Parties that form ex ante coalitions are not bound to stay together ex post. As the bargaining prior to the announcement of the results in the recent national election in India shows (see the Introduction), this assumption is quite plausible. To illustrate how ex ante behavior depends on the ex post bargaining protocol, we considered two (infinite horizon) protocols, the Austen-Smith and Banks procedure and the random recognition procedure. We characterized stationary equilibria in both cases, assuming externalities and compromise costs among players with different ideologies.

Our main focus was on the formation of ex ante coalitions. There are three reasons to form an ex ante coalition: to influence which government will form ex post, to manipulate the bargaining power within the government, and (with PV) to avoid splitting the vote. With the PV electoral system, ex ante coalitions between L and M increase their total seat shares. However, the division of seat shares induces a discontinuity, because $\mathrm{R}$ might withdraw from enough seats to give $\mathrm{M}$ its own majority. In this case, every ex ante agreement must give $\mathrm{M}$ all the benefits of being in government. With the ASB protocol ex post, ex ante coalitions might also form in order to manipulate the order in which players are recognized in the ex post protocol. Thus, with PR, although an ex ante coalition (joint list) between L and M does not increase their total vote shares, they may still form the joint list. Depending on the parameters, an MR coalition might instead form to ensure a majority for M. Although our model is simplistic (for example, there are only three parties), it illustrates the strategic interplay between legislative bargaining and seat-sharing arrangements in the settings of legislative elections.

Another key simplification is that all seats are ex ante identical. In reality, some districts 
might be "safe" for a party. For example, the former Indian Prime Minister Indira Gandhi came back to the Indian Parliament, after losing her own seat in 1977, by winning a byeelection in a "safe seat". It would be possible to model heterogeneity by considering districts in the set $[0,1]$, with an interval $[0, z(L)]$ representing the "safe area" for party L. The interval $[z(L), w(L)]$ would represent the seats that $\mathrm{L}$ would win in a three-way contest but lose if it were running solely against $M$. The remaining sub-interval is partitioned in a similar way. With this formulation, withdrawing from some seats is "free". For example, $\mathrm{R}$ can withdraw from any seat that it would not win by an outright majority, in order to

increase M's seat share. Party M would benefit from the fact that other parties would be more willing to withdraw in its favour. It would also be interesting to analyze the interaction in a Presidential system, which adds a key strategic player who may have veto power. These remain topics for future research.

\section{References}

[1] Austen-Smith, D., Banks, J. 1988. Elections, coalitions, and legislative outcomes. American Political Science Review 82, 405-422.

[2] Axelrod, R., 1970. The Conflict of Interest. Markham, Chicago.

[3] Bandyopadhyay, S., Chatterjee, K, 2006 Coalition theory and its applications: A Survey. Economic Journal, 116, 136-155

[4] Bandyopadhyay, S., Oak, M., 2004. Party formation and coalitional bargaining in a model of proportional representation. Discussion Paper. University of Birmingham, U.K.

[5] Bandyopadhyay, S., Oak, M. 2008. Coalition governments in a model of parliamentary democracy. The European Journal of Political Economy. 24(3), 554-56

[6] Baron, D., Ferejohn, J., 1989. Bargaining in legislatures. American Political Science Review 83, 1181-1206.

[7] Baron, D., Diermeier, D., 2001. Elections, governments, and parliaments under proportional representation. Quarterly Journal of Economics 116, 933-967

[8] Besley, T., Coate, S., 1997. An economic model of representative democracy. Quarterly Journal of Economics 112, 85-106.

[9] Blais, A , Indridason, I , 2007 Making Candidates Count: The Logic of Electoral Alliances in Two-Round Legislative Elections, The Journal of Politics, 69, 193-205 
[10] Chatterjee, K, Dutta, B, Ray, D and Sengupta, K, 1993. A noncooperative theory of coalitional bargaining, Review of Economic Studies, 60, 463-477.

[11] Debus, M, 2009 Pre-electoral commitment and government formation, Public Choice, $138,45-64$

[12] Dhillon, A., 2005. Political parties and coalition formation. group formation in economics. In: G Demange, G and M.Wooders, M. (Eds), Networks, Clubs and Coalitions, Cambridge University Press, New York, 289-311.

[13] Diermeier, D., Merlo, A., 2000. Government turnover in parliamentary democracies. Journal of Economic Theory 94, 46-79.

[14] Diermeier, D., Merlo, A., 2004. An empirical investigation of coalitional bargaining procedures. Journal of Public Economics 88, 783-797.

[15] Downs, A., 1957, An Economic Theory of Democracy. New York: Harper .

[16] Eraslan, H and Merlo, A, 2002. Majority rule in a stochastic model of bargaining, Journal of Economic Theory, Elsevier, 103 (1), 31-48

[17] Gallagher, K., Laver, K., Mair, P., 1995. Representative Government in Modern Europe. McGraw Hill, New York.

[18] Golder, S, 2006 a Pre-electoral coalition formation in parliamentary democracies, British Journal of Political Science 36, 193-212.

[19] Golder, S., 2006 b The Logic of Pre-Electoral Coalition Formation. Ohio State University Press

[20] Indridason, I., 2003. Coalitions and clientelism: A comparative study. Department of Political Science, University of Iceland.

[21] Indridason, I, 2005, A theory of coalitions and clientelism: coalition politics in Iceland 1945-2000, European Journal of Political Research, 44(3), 439-464

[22] Indridason, 2009, Proportional Representation, Majoritarian Legislatures and Coalitional Voting, mimeo, University of California Riverside

[23] Jackson, M., Moselle, B., 2002. Coalition and party formation in a legislative voting game. Journal of Economic Theory 103, 49-87. 
[24] Laver, K., Schofield, N., 1990. Multiparty Governments: The Politics of Coalitions in Europe. Oxford University Press, Oxford.

[25] Levy, G, 2004, A model of political parties. Journal of Economic theory, 115 (2),.250-277

[26] Morelli, M., 2004. Party formation and policy outcomes under different electoral systems. Review of Economic Studies 71, 829-853.

[27] Okada, A, 1996, A noncooperative coalitional bargaining game with random proposers. Games and Economic Behavior 16, 97-108

[28] Okada, A, 2007, Coalitional bargaining games with random proposers; theory and applications, mimeo Hitotsubashi University Graduate School of Economics, Tokyo, Japan.

[29] Osborne, M., Slivinski, A., 1996. A model of political competition with citizencandidates. Quarterly Journal of Economics 111, 65-96.

[30] Osborne, M., Tourky, R., 2002. Party formation in collective decision-making. Working paper, The University of Melbourne.

[31] Persson, T.,Roland, G. and Tabellini,G. 2007, Electoral rules and government spending in parliamentary democracies, Quarterly Journal of Political Science 2, 155-188.

[32] Pugh, M., 2002, The Making of Modern British Politics, 1867-1945, Blackwell.

[33] Ray, D, 2008, A Game-Theoretic Perspective on Coalition Formation, Oxford University Press, Oxford.

[34] Riker, W., 1962. The Theory of Political Coalitions. Yale University Press, New Haven, CT.

[35] Roemer, J., 2001. Political Competition: Theory and Applications. Harvard University Press, Cambridge, MA.

[36] Snyder J , Ting, M, 2002 An informational rationale for political parties, American Journal of Political Science, 46, 90-110

\section{Appendix}

\subsection{Stationary equilibria of the ASB bargaining game}

At the ex post stage, the parties bargain over government formation and surplus sharing. Each party's seat share $n(P)$ has been determined in the election and enters as a fixed term 
$\alpha n(P)$ in the payoff function. For simplicity, we omit this fixed term when deriving the payoff from the bargaining game.

Proposition 1 is proved via three Lemmas, corresponding to three different situations. Lemma 1 deals with the case where $\mathrm{M}$ is the smallest party, and so is the last party to make a proposal within each round. In this case, it is quite easy to show that the LM coalition is the equilibrium outcome.

Lemmas 2 and 3 deal with the case where $M$ is not the smallest party. Lemma 2 shows that if $\lambda=1$, then the LM coalition forms only if $S(M, R)<\frac{1}{3} S(L, M)$. Lemma 3 shows that when $\lambda=2$, the LM coalition must form. When $\lambda=2$, it takes some care to compute equilibrium payoffs, so the proof of Lemma 3 is slightly longer than the others.

Throughout, we restrict attention to stationary subgame perfect equilibria (SSPE). Let $s_{P}$ denote party P's equilibrium payoff. By stationarity, whenever any round is about to begin, $\mathrm{P}$ expects $s_{P}$. Recall that $\lambda$ denotes the number of periods $\mathrm{L}$ has to wait to make an offer after rejecting an offer from M.

Lemma 1 If $M$ is the smallest party, then the LM coalition must form. As $\delta \rightarrow 1$, M's payoff converges to

$$
\max \left\{\frac{\lambda}{3} S(L, M), S(M, R)\right\}
$$

Proof. Suppose M is the smallest party, so the protocol is either LRM or RLM. We first show that the LM coalition must form. Suppose, in order to derive a contradiction, that the equilibrium outcome is the MR coalition. Since M is the smallest party, M is the last to make an offer in each round. L will accept any offer from $\mathrm{M}$, since by rejecting, he expects to be left out in the next round (by stationarity). Therefore, $\mathrm{M}$ offers 0 to $\mathrm{L}$ which is accepted. Accordingly, $\mathrm{M}$ can reject all offers from $\mathrm{L}$ and $\mathrm{R}$ and eventually get all of the surplus in a coalition with $\mathrm{L}$. For $\delta$ close to 1 , this is better than forming a coalition with $\mathrm{R}$ (since $S(L, M)>S(M, R)$ ). This contradiction shows that the LM coalition must form in equilibrium.

Next, we compute M's equilibrium payoff $s_{M}$. If M makes an offer which is rejected, then a new round begins where (by stationarity) the LM coalition is expected to form, L's expected payoff is $s_{L}$, and R's expected payoff is negative (since it is left out). Therefore, $\mathrm{R}$ will accept any offer from $\mathrm{M}$, while $\mathrm{L}$ will accept any offer of at least $\delta s_{L}$. This implies that M's expected payoff when it is his turn to propose is

$$
\tilde{s}_{M}=\max \left\{S(L, M)-\delta s_{L}, S(M, R)\right\}
$$


When it is L's turn to propose, he will offer $\delta^{3-\lambda} \tilde{s}_{M}$ to $\mathrm{M}$ and $\mathrm{M}$ will accept. ${ }^{11}$ This implies that L's expected payoff when it is his turn to propose is

$$
\tilde{s}_{L}=S(L, M)-\delta^{3-\lambda} \tilde{s}_{M}
$$

If $\lambda=1$, so the protocol is LRM, then $s_{L}=\tilde{s}_{L}$. If $\lambda=2$, so the protocol is RLM, then (since the game starts with $\mathrm{R}$ making an offer which is rejected) $s_{L}=\delta \tilde{s}_{L}$. Thus, in either case,

$$
s_{L}=\delta^{\lambda-1} \tilde{s}_{L}=\delta^{\lambda-1}\left(S(L, M)-\delta^{3-\lambda} \tilde{s}_{M}\right)
$$

Substituting (25) in (23) and taking the limit as $\delta \rightarrow 1$ yields

$$
\tilde{s}_{M}=\max \left\{\frac{1-\delta^{\lambda}}{1-\delta^{3}} S(L, M), S(M, R)\right\} \rightarrow \max \left\{\frac{\lambda}{3} S(L, M), S(M, R)\right\}
$$

M's equilibrium payoff $s_{M}$ converges to the same limit because he is offered $\delta^{3-\lambda} \tilde{s}_{M}$ by L.

Lemma 2 Suppose the protocol is either $M L R$ or $R M L$. If $S(M, R)<S(L, M) / 3$ then for $\delta$ close to 1 , the $L M$ coalition forms. If $S(M, R)>S(L, M) / 3$ then for $\delta$ close to 1 , the $M R$ coalition forms. In either case, as $\delta \rightarrow 1, M$ 's payoff converges to

$$
\max \left\{\frac{1}{3} S(L, M), S(M, R)\right\}
$$

Proof. (i) Consider the protocol MLR. If M rejects an offer from L, then M expects to get $\delta^{2} s_{M}$. To see this, note that $\mathrm{R}$ is the next to propose. Either R's offer is rejected, in which case $\mathrm{M}$ has to wait and get $s_{m}$ when the new round starts. Or else M accepts an offer from $\mathrm{R}$, but this offer cannot exceed $\delta s_{M}$, by a standard argument (if it exceeds $\delta s_{M}$ then R could lower the offer slightly and it would still be accepted).

When it is L's turn to make a proposal, he will offer $\delta^{2} s_{M}$ to $\mathrm{M}$ and, again by a standard argument, this must be accepted by $\mathrm{M}$ (for L could surely get acceptance by offering $\delta^{2} s_{M}+\varepsilon$ which is feasible). Continuing the same argument, when it is M's turn to make an offer, L will accept any offer which gives him at least $\delta\left(S(L, M)-\delta^{2} s_{M}\right)$. R will accept even a zero

\footnotetext{
${ }^{11}$ This is obvious if the protocol is RLM, since then $\lambda=2$ and M is the next to make an offer after L. If $\lambda=1$, so the protocol is LRM, then $\mathrm{R}$ makes the next offer after L. But, even if $\mathrm{R}$ can make an acceptable offer to M, R will never offer more than $\delta \tilde{s}_{M}$. Therefore, L can offer $\delta^{2} \tilde{s}_{M}$.
} 
offer, since a rejection means $\mathrm{L}$ makes an offer to $\mathrm{M}$ which is accepted. Therefore,

$$
s_{M}=\max \left\{S(L, M)-\delta\left(S(L, M)-\delta^{2} s_{M}\right), S(M, R)\right\}
$$

which is equivalent to

$$
s_{M}=\max \left\{\frac{1-\delta}{1-\delta^{3}} S(L, M), S(M, R)\right\} \rightarrow \max \left\{\frac{1}{3} S(L, M), S(M, R)\right\}
$$

If $S(M, R)>S(L, M) / 3$, then for $\delta$ close to one, $S(M, R)>\frac{1-\delta}{1-\delta^{3}} S(L, M)$. This means M's offer goes to R. If $S(M, R)<S(L, M) / 3$, then for $\delta$ close to one, $S(M, R)<\frac{1-\delta}{1-\delta^{3}} S(L, M)$ and M's offer goes to L.

(ii) If the protocol is RML, the argument is similar and is omitted.

Lemma 3 Suppose the protocol is MRL or LMR. For $\delta$ close to 1, the LM coalition forms. As $\delta \rightarrow 1$, M's payoff converges to

$$
\max \left\{\frac{2}{3} S(L, M), S(M, R)\right\}
$$

Proof. (i) Consider the protocol MRL, where M is formateur. First, we show the LM coalition must form. Suppose - in order to obtain a contradiction - that M offers a payoff of $w \geq 0$ to $\mathrm{R}$, which is accepted. This implies that if $\mathrm{M}$ rejects an offer from $\mathrm{L}, \mathrm{M}$ expects to get $S(M, R)-w$ next period (the beginning of the new round). Therefore, $\mathrm{L}$ will offer him $\delta(S(M, R)-w)$, and this is accepted by $\mathrm{M}$. Therefore, if $\mathrm{M}$ rejects an offer from $\mathrm{R}, \mathrm{M}$ will get $\delta(S(M, R)-w)$ in the next period. Hence $\mathrm{R}$ offers $\delta^{2}(S(M, R)-w)<S(M, R)$, and $\mathrm{M}$ accepts. But then L must accept any offer from M, since if he rejects he its left out. Clearly, $\mathrm{M}$ prefers to offer 0 to $\mathrm{L}$ rather than $w$ to $\mathrm{R}$, a contradiction. Thus, in each round, M makes an offer to $\mathrm{L}$, not to $\mathrm{R}$.

Having established that the LM coalition forms, it remains to show that M's payoff takes the familiar form.

$\mathrm{M}$ is the formateur. In equilibrium, he offers a payoff of $s_{L}$ to $\mathrm{L}$, which is accepted. $\mathrm{M}$ himself gets $s_{M}=S(L, M)-s_{L}$. If $\mathrm{M}$ rejects an offer from $\mathrm{L}$, next period (which is the beginning of the next round), $\mathrm{M}$ gets $s_{M}$. Therefore, $\mathrm{L}$ will offer $\delta s_{M}$, and $\mathrm{M}$ accepts. Therefore, if $\mathrm{M}$ rejects an offer by $\mathrm{R}, \mathrm{M}$ will get $\delta s_{M}$ in the next period. Hence M must accept an offer of $\delta^{2} s_{M}+\varepsilon$ from $\mathrm{R}$. If $\delta^{2} s_{M}<S(M, R)$, then such offer is feasible, and $\mathrm{R}$ is sure to make a proposal to $\mathrm{M}$ that will be accepted. In this case, $\mathrm{L}$ will expect to be left out 
if he rejects an offer from $\mathrm{M}$, hence $\mathrm{L}$ will accept any offer, which means $s_{M}=S(M, R)$. For $\delta$ close to 1 , this contradicts $\delta^{2} s_{M}<S(M, R)$. This contradiction shows we must have

$$
\delta^{2} s_{M} \geq S(M, R)
$$

There are two possibilities.

Case $\boldsymbol{\alpha}$. Suppose there is strict inequality in (27). Then, R cannot make any proposal to $\mathrm{M}$ that $\mathrm{M}$ will accept. Hence, if $\mathrm{L}$ rejects $\mathrm{M}$ 's offer, $\mathrm{R}$ cannot make any agreement with $\mathrm{M}$, and $\mathrm{L}$ will offer $\delta s_{M}$ two periods later, which M accepts. Thus, L will accept M's offer if and only if it exceeds $\delta^{2}\left(S(L, M)-\delta s_{M}\right)$. This expression must equal $s_{L}=S(L, M)-s_{M}$, which implies

$$
s_{M}=\frac{1-\delta^{2}}{1-\delta^{3}} S(L, M)
$$

For $\delta$ close to $1,(28)$ is consistent with $(27)$ if $S(M, R)<\frac{2}{3} S(L M)$, but not if $S(M, R)>$ $\frac{2}{3} S(L M)$. Thus, case $\alpha$ cannot happen if $S(M, R)>\frac{2}{3} S(L M)$. Suppose instead $S(M, R)<$ $\frac{2}{3} S(L M)$. M offers $s_{L}=S(L, M)-s_{M}$ to L, and L accepts any offer of at least this much. If M's offer is rejected, in the next period M rejects all feasible offers from $\mathrm{R}$. This is optimal for M, because

$$
S(M, R)<\delta^{2} s_{M}=\delta^{2} \frac{1-\delta^{2}}{1-\delta^{3}} S(L M)
$$

for $\delta$ close to 1 . L offers $\delta s_{M}$ to $\mathrm{M}$, and $\mathrm{M}$ accepts any offer of at least this much. These strategies form an SSPE, and $s_{M} \rightarrow \frac{2}{3} S(L M)$.

Case $\boldsymbol{\beta}$. Suppose there is equality in $(27)$, so

$$
s_{M}=\frac{1}{\delta^{2}} S(M, R)
$$

In any round, $\mathrm{M}$ offers $s_{L}=S(L, M)-s_{M}$ to $\mathrm{L}$, and $\mathrm{L}$ accepts any offer of at least this much. If M's offer is rejected, then in the next period $\mathrm{R}$ offers $S(M, R)$ to $\mathrm{M}$. When $\mathrm{M}$ gets this offer, (29) implies that he is indifferent between accepting and rejecting. In order to give $\mathrm{L}$ an incentive to accept $s_{L}$ but nothing less than that, M's decision must depend on the offer L rejected. ${ }^{12}$ If $\mathrm{L}$ rejected an offer of $s_{L}$ or more, then $\mathrm{M}$ "punishes" L by accepting $S(M, R)$ from $\mathrm{R}$. But if $\mathrm{L}$ rejected an offer of strictly less than $s_{L}$, then $\mathrm{M}$ rejects R's offer. If R's offer is rejected, L offers $\delta s_{M}$ to $\mathrm{M}$, and $\mathrm{M}$ accepts any offer of at least this much.

With these strategies, L clearly prefers to accept an offer of $s_{L}$. We also need to verify that he prefers to reject offers strictly below $s_{L}$. If L rejects an offer of $s_{L}-\varepsilon$, he must wait for two periods and then makes an offer of $\delta s_{M}$ to M which M accepts. Thus, L rejects $s_{L}-\varepsilon$

\footnotetext{
${ }^{12}$ This does not contradict stationarity, since behavior does not depend on events in any previous round.
} 
for any $\varepsilon>0$ if

$$
s_{L}=S(L, M)-s_{M} \leq \delta^{2}\left[S(L, M)-\delta s_{M}\right]
$$

This inequality holds for $\delta$ close to 1 if $S(M, R)>\frac{2}{3} S(L M)$ but is violated if $S(M, R)<$ $\frac{2}{3} S(L M)$ (using (29)). Thus, case $\beta$ can only happen in the former case. M's payoff is given by (29) and converges to $S(M, R)$.

To summarize, if $S(M, R)<\frac{2}{3} S(L M)$ then there is a Case $\alpha$ SSPE where $s_{M} \rightarrow \frac{2}{3} S(L M)$. If $S(M, R)>\frac{2}{3} S(L M)$ then there is a Case $\beta$ SSPE where $s_{M} \rightarrow S(M, R)$.

(ii) If the protocol is LMR, then it is obvious that the LM coalition forms, since L is the formateur. The rest of the argument is similar to case (i), and is omitted.

\subsection{Stationary equilibria with random recognition}

Recall that $\phi_{P}$ denotes the probability that player $P$ is recognized in any period. Also recall that proposals specify non-negative shares of the rents from office. There are no sidepayments outside this. Therefore, the smallest amount a player can get in an ex post coalition is 0 . Let $\widetilde{s}_{P}$ denote the expected continuation payoff for party $P$, and $s_{P} \equiv \max \left\{0, \widetilde{s}_{P}\right\}$.

Proposition 12 There is a unique SSPE in the post-election coalition formation game with repeated random recognition. As $\delta \rightarrow 1$, the LM government always forms, and limit SSPE payoffs are as follows. If

$$
S(M R)<\frac{\phi_{M}}{\phi_{L}+\phi_{M}} S(L M)
$$

then

$$
\begin{aligned}
& s_{M}=\frac{\phi_{M}}{\phi_{L}+\phi_{M}} S(L M) \\
& s_{L}=\frac{\phi_{L}}{\phi_{L}+\phi_{M}} S(L M)
\end{aligned}
$$

and

$$
\widetilde{s}_{R}=-x_{R}(L M) .
$$

If

$$
S(M R)>\frac{\phi_{M}}{\phi_{L}+\phi_{M}} S(L M)
$$

then $s_{M}=S(M R), s_{L}=S(L M)-S(M R)$, and $\widetilde{s}_{R}=-x_{R}(L M)$. 
Proof. In equilibrium, $\mathrm{R}$ is either relevant or irrelevant. First, suppose $\mathrm{R}$ is irrelevant, that is, $L$ and $M$ make offers to each other and any offer made by $R$ is rejected. The only agreement, whenever there is one, is between $L$ and $M$. Thus $s_{R}=0$. To see if this can be an equilibrium, we calculate the minimum amount that needs to be paid to induce acceptance by the player concerned (with the usual argument that in equilibrium each player accepts if indifferent). For $P \in\{L, M\}$, this minimum must be $\delta$ times the continuation payoff $s_{P}$, which is positive because they do not expect to be outside the government. Thus, in each round, with probability $\phi_{L}$ player L makes an offer of $\delta s_{M}$ to $\mathrm{M}$, with probability $\phi_{M}$ player $\mathrm{M}$ makes an offer of $\delta s_{L}$ to $\mathrm{L}$, and with probability $\phi_{R}$ the game moves to the next round. Accordingly, the equilibrium payoffs must satisfy the following equations:

$$
\begin{aligned}
s_{L} & =\phi_{L}\left(S(L, M)-\delta s_{M}\right)+\phi_{M} \delta s_{L}+\phi_{R} \delta s_{L} \\
s_{M} & =\phi_{L} \delta s_{M}+\phi_{M}\left(S(L, M)-\delta s_{L}\right)+\phi_{R} \delta s_{M} \\
\widetilde{s}_{R} & =\phi_{L}\left(-x_{R}(L, M)\right)+\phi_{M}\left(-x_{R}(L, M)+\phi_{R} \delta \widetilde{s}_{R} .\right.
\end{aligned}
$$

From the first two equations we get

$$
s_{M}=\frac{\phi_{M} S(L, M)(1-\delta)}{\left(1-\delta+\delta \phi_{M}+\delta \phi_{L}\right)(1-\delta)}=\frac{\phi_{M} S(L, M)}{\left(1-\delta+\delta \phi_{M}+\delta \phi_{L}\right)} .
$$

As $\delta \rightarrow 1, s_{M} \rightarrow \frac{\phi_{M}}{\phi_{M}+\phi_{L}} S(L, M)$, as in (31), and (32) is obtained similarly. If the inequality (30) holds, then there is not enough surplus in the MR coalition for M to consider joining it, and $\mathrm{R}$ is truly irrelevant. The equilibrium payoffs are given by (31), (32) and (33). But the equilibrium breaks down if the inequality (34) holds, because $\mathrm{R}$ could offer $\mathrm{M}$ more than $\frac{\phi_{M}}{\phi_{M}+\phi_{L}} S(L, M)$.

Now suppose inequality (34) holds, so the above is not an equilibrium. In this case, $\mathrm{R}$ cannot be irrelevant. We consider candidate stationary equilibria of the following kinds: (i) A pure strategy equilibrium with all offers being accepted and $s_{L}>0$; (ii) Mixed strategies in making offers and (iii) mixed strategies in responses to offers. The stationarity requirement ensures that the continuation payoffs are the same in every period.

Condition (i) in the preceding paragraph gives us

$$
\begin{aligned}
s_{L} & =\phi_{L}\left(S(L, M)-\delta s_{M}\right)+\phi_{M} \delta s_{L}+\phi_{R}\left(-x_{L}(M, R)\right), \\
s_{M} & =\phi_{L}\left(\delta s_{M}\right)+\phi_{M}\left(S(L, M)-\delta s_{L}\right)+\phi_{R} \delta s_{M}, \\
\widetilde{s}_{R} & =\phi_{R}\left(S(M, R)-\delta s_{M}\right)+\left(1-\phi_{R}\right)\left(-x_{R}(L, M)\right) .
\end{aligned}
$$


As $\delta \rightarrow 1$, these expressions, when simplified, give us

$$
s_{M}=\frac{\phi_{L}+\phi_{R}}{\phi_{R}} S(L, M)+\phi_{M} \phi_{R} x_{L}(M, R) .
$$

But $s_{M}$ cannot be greater than $S(L, M)$, so equation (35) cannot hold. Therefore $s_{L}$ cannot be assumed to be strictly positive but must be 0 . But then $M$ can obtain $S(L, M)$ from making an offer to $L$ and will not accept an offer from $R$, which contradicts our assumption. (The value of $s_{R}$ does not play any role in the above calculation.) Therefore (i) is ruled out as a candidate stationary equilibrium.

We consider (ii) above. Since $L$ and $R$ have only one option each, it must be $M$ who randomizes (between offers to $L$ and $R$ ). By an analysis similar to the above, we can show that this is impossible (the assumed probability with which $M$ chooses $L$ turns out to have a negative value, which is a contradiction). The only possible stationary equilibrium therefore must be of the form (iii). Here again, it must be $M$ who randomizes between accepting and rejecting an offer. This offer must be either $S(L, M)$ from $L$ or $S(M, R)$ from $R$; for otherwise, the proposer could deviate and offer $\varepsilon$ more, thus breaking the indifference and forcing $\mathrm{M}$ to accept with probability 1 . But an offer of $S(L, M)$ from $L$ is clearly not part of any equilibrium (an offer of $\delta S(L, M)$ would be acceptable and a profitable deviation for $L$, since $s_{M} \leq S(L, M)$ ). Thus, the only possible equilibrium of this kind must have $\mathrm{R}$ offering all the surplus $S(M, R)$ to M. Player M accepts this offer with probability $y$, where $0<y<1$.

In this (history independent) equilibrium, $\mathrm{M}$ is indifferent between accepting and rejecting:

$$
\delta s_{M}=S(M, R)
$$

Therefore $M$ must make an acceptable offer to $L$, when it is his turn to do so, since $S(M, R) / \delta$ is infeasible for the MR coalition. We can write down the equations as before, to describe the equilibrium expected payoffs. Once again $R$ 's payoffs are not important for the purpose of calculating the payoffs of the others:

$$
\begin{aligned}
s_{L} & =\phi_{L}(S(L, M)-S(M, R))+\phi_{M} \delta s_{L}+\phi_{R}\left[y\left(-x_{L}(M, R)\right)+(1-y) \delta s_{L}\right], \\
s_{M} & =\frac{S(M, R)}{\delta}=\phi_{L} S(M, R)+\phi_{M}\left(S(L, M)-\delta s_{L}\right)+\phi_{R} S(M, R) .
\end{aligned}
$$

The second equation gives

$$
\delta^{2} \phi_{M} s_{L}=\delta\left(1-\phi_{M}\right) S(M, R)-S(M, R)+\delta \phi_{M} S(L, M)
$$


or

$$
\delta s_{L}=\frac{\delta \phi_{M} S(L, M)-\left(1-\delta\left(1-\phi_{M}\right)\right) S(M, R)}{\delta \phi_{M}}
$$

From these expressions, we can calculate the value of $y$ as below.

$$
\left(\delta^{2} \phi_{R} s_{L}+\delta \phi_{R} x_{L}\right) y=\delta \phi_{L}[S(L, M)-S(M, R)]-\delta s_{L}\left(1-\delta+\delta \phi_{L}\right)
$$

When $\delta=1, s_{L}=S(L, M)-S(M, R)$ and substituting this in the expression above, we get $y=0$ when $\delta=1$. We now wish to show that $y>0$ for $\delta<1$ but close to 1 , so that the equilibrium exists for $\delta<1$. We therefore differentiate both sides of the expression above with respect to $\delta$ and evaluate the derivative at $\delta=1$. We first note that $\frac{\partial s_{L}}{\partial \delta}$ is positive at $\delta=1$. Differentiating and setting $\delta=1$, we get

$$
2 \phi_{M} s_{L}+\phi_{M} \frac{\partial s_{L}}{\partial \delta}=\phi_{M}[S(L, M)-S(M, R)]+S(M, R)
$$

or

$$
\begin{aligned}
\frac{\partial s_{L}}{\partial \delta} & =\frac{S(M, R)}{\phi_{M}}-[S(L, M)-S(M, R)] \\
& >\frac{1}{\phi_{M}+\phi_{L}}\left(1+\phi_{M}\right) S(L, M)-S(L, M)=\frac{S(L, M)}{\phi_{M}+\phi_{L}}\left(1-\phi_{L}\right)>0
\end{aligned}
$$

where the first inequality uses (34). We now differentiate both sides of (36) with respect to $\delta$ and evaluate again at $\delta=1$, where we know $y=0$. This gives us, using the above expression for $\frac{\partial s_{L}}{\partial \delta}$

$$
\begin{aligned}
& y\left(2 \delta \phi_{R} s_{L}+\phi_{R} x_{L}+\delta^{2} \phi_{R} \frac{\partial s_{L}}{\partial \delta}\right)+\left(\delta^{2} \phi_{R} s_{L}+\delta \phi_{R} x_{L}\right) \frac{\partial y}{\partial \delta} \\
= & \phi_{L}[S(L, M)-S(M, R)]-\delta s_{L}\left(-1+\phi_{L}\right)-s_{L}\left(1-\delta+\delta \phi_{L}\right)-\delta\left(1-\delta+\delta \phi_{L}\right) \frac{\partial s_{L}}{\partial \delta} \\
= & \left(\phi_{R} s_{L}+\phi_{R} x_{L}\right) \frac{\partial y}{\partial \delta}=s_{L}\left(1-\phi_{L}\right)-\phi_{L} \frac{\partial s_{L}}{\partial \delta}=s_{L}\left(1-\phi_{L}\right)-\phi_{L}\left(\frac{S(M, R)}{\phi_{M}}-s_{L}\right) \\
= & S(L, M)\left[1-\phi_{L}+\phi_{L}\right]-S(M, R)\left[1-\phi_{L}+\frac{\phi_{L}}{\phi_{M}}+\phi_{L}\right] \\
= & S(L, M)-S(M, R) \frac{\phi_{M}+\phi_{L}}{\phi_{M}}<0
\end{aligned}
$$

by the inequality (34). Therefore $\frac{\partial y}{\partial \delta}<0$ at $\delta=1$, so if $\delta$ decreases below $1, y$ increases to $y>0$, as was to be shown.

It may be remarked that non-stationary pure strategy equilibria exist as well. Suppose 
(34) applies and consider a non-stationary equilibrium of the following form. M offers $\delta s_{L}$ to $\mathrm{L}, \mathrm{L}$ offers $\delta s_{M}$ to $\mathrm{M}$ and $\mathrm{R}$ offers $S(M, R)$ to $\mathrm{M}$, where

$$
s_{M}=\phi_{M}\left(S(L, M)-\delta s_{L}\right)+\phi_{L} \delta s_{m}+\phi_{R} S(M, R)
$$

so

$$
s_{M}=\frac{\phi_{M}}{1-\phi_{L} \delta}\left(S(L, M)-\delta s_{L}\right)+\frac{\phi_{R}}{1-\phi_{L} \delta} S(M, R)
$$

These offers are all accepted with probability one. L's payoff is

$$
s_{L}=\phi_{L}\left(S(L, M)-\delta s_{M}\right)+\phi_{M} \delta s_{L}-\phi_{R} x_{L}(M, R)
$$

SO

$$
s_{L}=\frac{\phi_{L}}{\left(1-\phi_{M} \delta\right)}\left(S(L, M)-\delta s_{M}\right)-\frac{\phi_{R}}{\left(1-\phi_{M} \delta\right.} x_{L}(M, R)
$$

These two equations can be solved for $s_{m}$ and $s_{L}$. At $\delta=1$, the expressions for $s_{M}$ and $s_{L}$ are as follows:

$$
\begin{aligned}
s_{M} & =\phi_{M}\left[S(L, M)+x_{L}\right]+\left(1-\phi_{M}\right) S(M, R), \\
s_{L} & =\phi_{L}\left[S(L, M)-s_{M}\right]-\phi_{R} x_{L}(M, R) .
\end{aligned}
$$

This is feasible if $s_{L} \geq 0$, that is, $x_{L}$ is not too high and $s_{M}$ does not exceed $S(L, M)$. Here $M$ can get strictly more than in the stationary equilibrium described earlier, provided he accepts $R$ 's offer (of less than his equilibrium payoff). This is therefore the only deviation we need to check, i.e. for M to reject R's offer. If he does, the continuation payoffs and the strategies are now given by the stationary equilibrium above, rather than by (37). Thus, M's continuation payoff if he rejects $R$ 's offer is $S(M, R)$, so he accepts the offer.

\subsection{Ex ante coalitions when $\mathbf{R}$ is ex post relevant under Plurality Voting and ASB}

Here, we give a more detailed consideration of what kind of coalitions are formed when $\mathrm{R}$ is ex post relevant under Plurality Voting and ASB. In this case, $\mathrm{R}$ may also be able to participate in a viable ex ante coalition that does not give $\mathrm{M}$ its own majority, by transferring seats to $\mathrm{M}$ in a way which changes $\lambda$ and hence M's share of the surplus. Recall that M cannot transfer seats to $\mathrm{R}$, so any ex ante agreement between $\mathrm{M}$ and $\mathrm{R}$ to change $\lambda$ must have $\mathrm{M}$ getting seats from $\mathrm{R}$. To avoid tedious repetition, we only focus on the case where $S(M, R)>\frac{1}{3} S(L, M)$ and $w(M)>w(R)>w(L)$. (The other cases can be worked out 
similarly). If there is no ex ante agreement, then $\lambda=2$ so the LM coalition forms ex post.

As before, the MR coalition with $\mathrm{M}$ getting its own majority can occur if

$$
x_{R}(L M)-x_{R}(M)>\alpha w(R) \frac{\frac{1}{2}-w(M)}{1-z(L)-w(M)} .
$$

If this is violated, the MR coalition might still form ex ante in order to produce the MR coalition ex post. Recall that the MR coalition forms ex post if $\mathrm{L}$ is not the formateur and $\lambda=1$. This can be achieved by making sure that $\mathrm{R}$ has fewer seats than $\mathrm{L}$, so the protocol becomes MLR (with $\lambda=1$ ) instead of MRL. Let $\mathrm{R}$ drop out of $r$ seats. Then M gets $(1-r) w(M)+r(1-z(L))$ seats, $\mathrm{L}$ gets $(1-r) w(L)+r z(L), \mathrm{R}$ gets $(1-r) w(R)$. We require $\mathrm{L}$ to get more seats than $\mathrm{R}$, that is,

$$
(1-r) w(L)+r z(L) \geq(1-r) w(R)
$$

which is the same as

$$
r \geq \frac{w(R)-w(L)}{w(R)-w(L)+z(L)}
$$

R's payoff will be $\alpha(1-r) w(R)$. For $\mathrm{R}$ to be willing to participate, we need

$$
\alpha(1-r) w(R) \geq \alpha w(R)-x_{R}(L, M)
$$

which is the same as

$$
r \leq \frac{x_{R}(L, M)}{\alpha w(R)}
$$

Thus, such agreement is feasible if

$$
\frac{w(R)-w(L)}{w(R)-w(L)+z(L)} \leq r \leq \frac{x_{R}(L, M)}{\alpha w(R)}
$$

M's share has to be less than $1 / 2$,

$$
(1-r) w(M)+r(1-z(L)) \leq \frac{1}{2}
$$

which is

$$
r \leq \frac{\frac{1}{2}-w(M)}{1-z(L)-w(M)}
$$

M's payoff is increasing in $r$ so he prefers the biggest $r$, which is

$$
r=\min \left\{\frac{x_{R}(L, M)}{\alpha w(R)}, \frac{\frac{1}{2}-w(M)}{1-z(L)-w(M)}\right\},
$$


assuming

$$
\min \left\{\frac{x_{R}(L, M)}{\alpha w(R)}, \frac{\frac{1}{2}-w(M)}{1-z(L)-w(M)}\right\} \geq \frac{w(R)-w(L)}{w(R)-w(L)+z(L)}
$$

M's payoff will then be

$\alpha((1-r) w(M)+r(1-z(L)))+S(M, R)=\alpha \min \left\{\frac{1}{2}, w(M)+\frac{x_{R}(L, M)}{\alpha w(R)}(1-z(L)-w(M))\right\}+S(M, R)$

However, notice that the LM coalition could also form ex ante in order to avoid splitting the vote. Suppose L and M make an ex ante agreement, M drops out of $m$ and L of $\ell$ districts. $\mathrm{M}$ will get $\ell(1-z(R))+(1-m-\ell) w(M)$, L will get $m(1-z(R))+(1-m-\ell) w(L)$, R gets $(m+\ell) z(R)+(1-m-\ell) w(R)$. Suppose L does not move ahead of $\mathrm{R}$, so $\lambda=2$ is constant. Then, M's payoff is

$$
\alpha[\ell(1-z(R))+(1-m-\ell) w(M)](1-z(R))+\frac{2}{3} S(L, M)
$$

L will only agree if

$$
m(1-z(R))+(1-m-\ell) w(L) \geq w(L)
$$

In addition, since $\mathrm{L}$ cannot be bigger than $\mathrm{R}$,

$$
m(1-z(R))+(1-m-\ell) w(L) \leq(m+\ell) z(R)+(1-m-\ell) w(R)
$$

So the best $\lambda=2$ preserving agreement for $\mathrm{M}$ maximizes his payoff subject to these two constraints. If $\mathrm{M}$ makes a take-it-or-leave-it offer (and the condition for $\mathrm{R}$ agreeing to give $\mathrm{M}$ its own majority is violated), that is the coalition that will form. Note, where $S(M R) \geq \frac{2}{3} S(L M) \lambda$ does not affect the division of surplus though it can affect which coalition can form ex post. 